Aus der Klinik für Neurochirurgie

(Prof. Dr. med. V. Rohde)

der Medizinischen Fakultät der Universität Göttingen

\title{
Gibt es Parameter, die den Erfolg einer Minibohrlochtrepanation eines chronischen Subduralhämatoms
} vorhersagen?

\author{
INAUGURAL-DISSERTATION \\ zur Erlangung des Doktorgrades \\ für Zahnheilkunde \\ der Medizinischen Fakultät der \\ Georg-August-Universität zu Göttingen
}

vorgelegt von

Luisa Hoederath (geb. Baurmann)

aus

Menden/Sauerland

Göttingen 2018 
Dekan:

I. Berichterstatter

II. Berichterstatter

Datum der mündlichen Prüfung:
Prof. Dr. rer.nat. H. K. Kroemer

Prof. Dr. med. Veit Rohde

PD Dr. med. Caspar Stephani

18.02.2019 
Hiermit erkläre ich, die Dissertation mit dem Titel "Gibt es Parameter, die den Erfolg einer Minibohrlochtrepanation eines chronischen Subduralhämatoms vorhersagen?“ eigenständig angefertigt und keine anderen als die von mir angegebenen Quellen und Hilfsmittel verwendet zu haben.

Göttingen, den 


\section{Inhaltsverzeichnis}

Abbildungsverzeichnis ..................................................................................... III

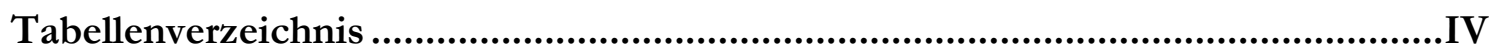

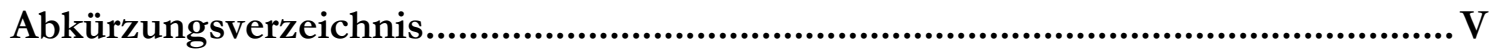

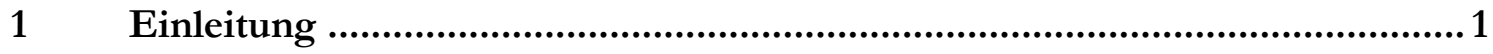

$1.1 \quad$ Inzidenz

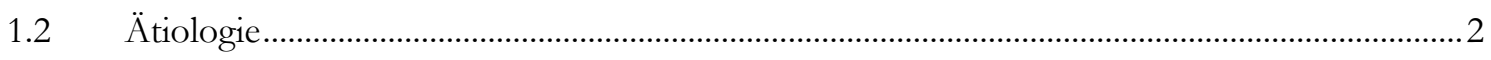

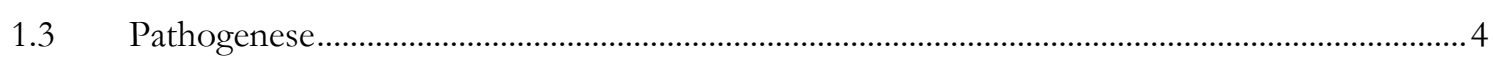

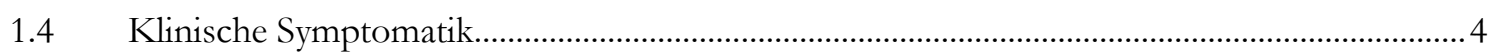

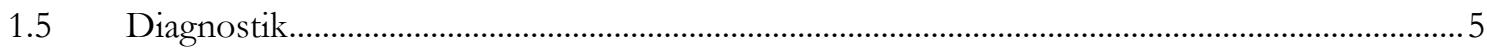



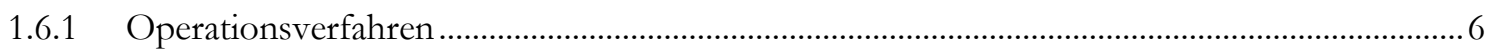





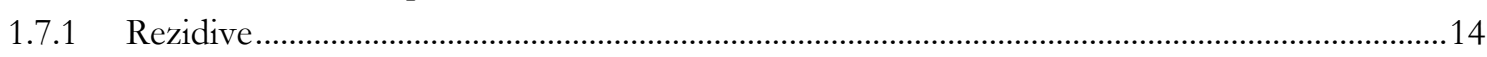

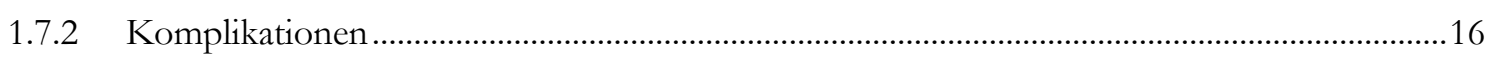

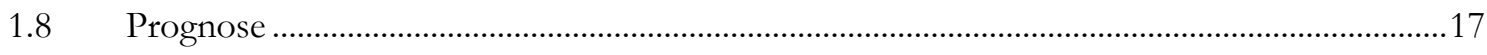

$2 \quad$ Fragestellung und Ziel der Studie ........................................................... 18



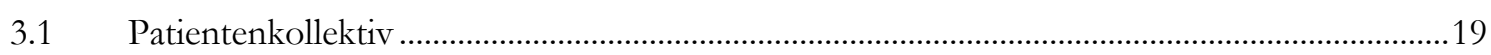

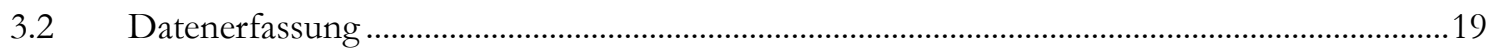

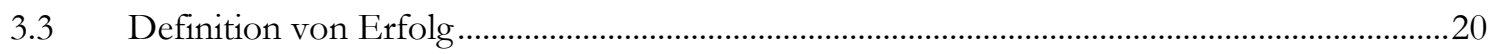

3.4 Einteilung der Patienten nach Karnofsky-Index.................................................................22

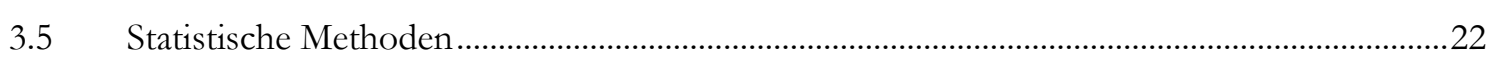

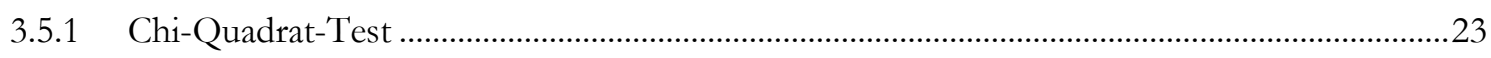

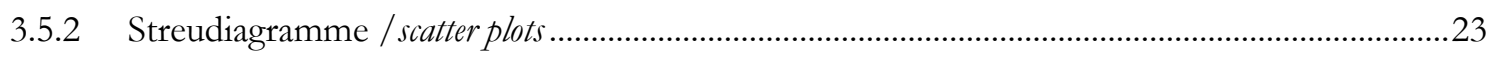

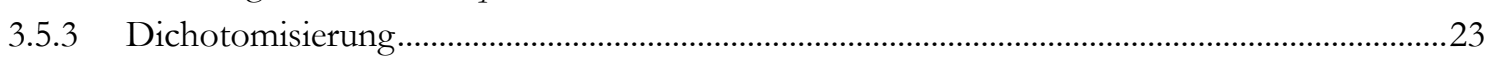

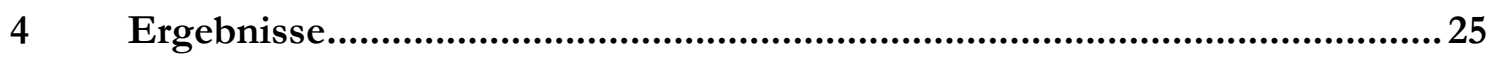

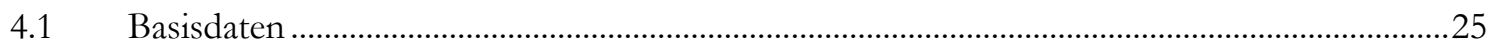

4.2 Daten zu Patienten mit Therapieerfolg..................................................................................2

4.3 Auswertung der Korrelation zwischen nötigen Reoperationen und Therapieerfolg ..............36

4.4 Daten zu Patienten mit Therapieversagen/nötigem Wechsel auf invasivere

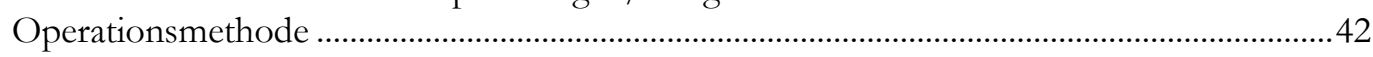

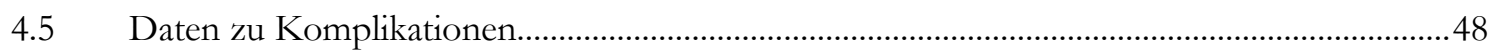




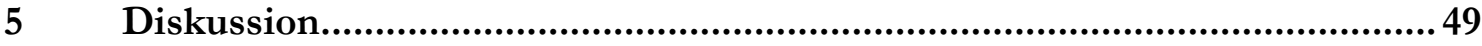

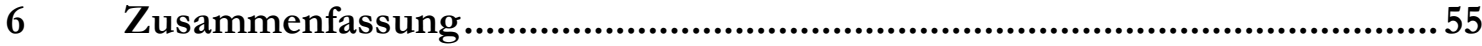

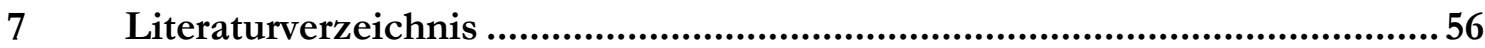




\section{Abbildungsverzeichnis}

Abbildung 1: Computertomografische Darstellung eines beidseitigen cSDH 5

Abbildung 2: Instrumentarium für die Minibohrlochtrepanation (Drillbohrertrepanation)

Abbildung 3: Minibohrlochtrepanation, Markierung der Trepanationsstelle ...........................7

Abbildung 4: Minibohrlochtrepanation, Lokalanästhesie ...................................................... 7





Abbildung 7: Minibohrlochtrepanation, Hämatomdrainage …............................................... 8

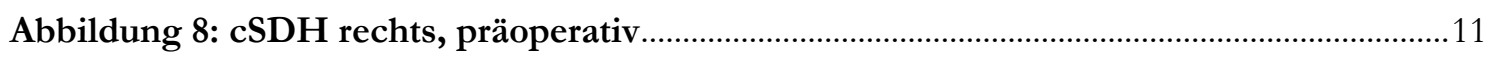

Abbildung 9: cSDH rechts, Erstkontrolle postoperativ ............................................................11

Abbildung 10: cSDH rechts, Verlaufskontrolle am 4. postoperativen Tag ..............................12

Abbildung 11: cSDH rechts, Verlaufskontrolle am 16. postoperativen Tag ..............................12

Abbildung 12: cSDH rechts, Verlaufskontrolle 6 Wochen postoperativ ....................................13

Abbildung 13: Verlaufskontrolle 14 Wochen postoperativ mit vollständiger Heilung des



Abbildung 14: Übersichtsdiagramm zur Datenerhebung .......................................................21 


\section{Tabellenverzeichnis}

Tabelle 1: Geschlechter- und Altersverteilung innerhalb des Patientenkollektivs ...................25

Tabelle 2: Verteilung der Leukozyten-, Thrombozyten- und Hämoglobinwerte.......................25



Tabelle 4: Verteilung der präoperativen Antikoagulanzien .........................................................27

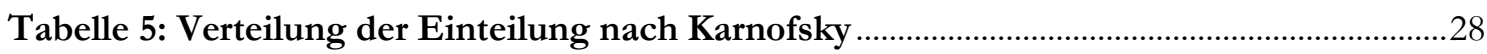

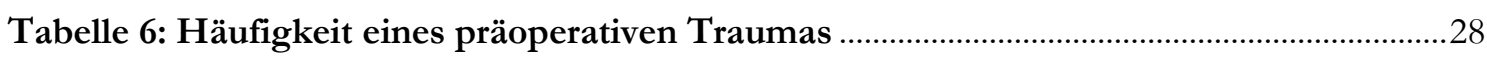

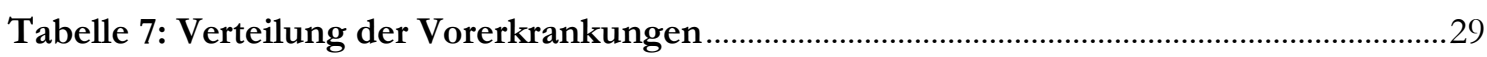

Tabelle 8: Therapieerfolg und -versagen innerhalb des Patientenkollektivs ............................29

Tabelle 9: Anzahl Minibohrlochtrepanationen unter Therapieerfolgen ........................................30

Tabelle 10: Therapieerfolg und -versagen unter Patienten mit alleiniger

Minibohrlochtherapie ......................................................................................................... 30

Tabelle 11: Altersverteilung der Patienten mit Therapieerfolg und -versagen............................ 31

Tabelle 12: Verteilung der Blutwerte unter den Patienten mit Therapieerfolg ...........................31

Tabelle 13: Verteilung der Gerinnungswerte unter den Patienten mit Therapieerfolg ............32

Tabelle 14: Verteilung der Antikoagulanzien unter den Patienten mit Therapieerfolg ...........33

Tabelle 15: Häufigkeit eines präoperativen Traumas unter Patienten mit

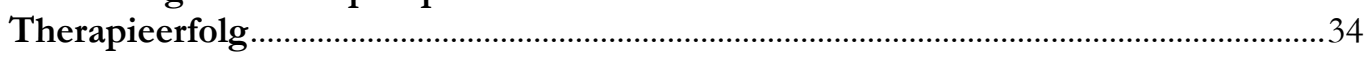

Tabelle 16: Bewertung nach Karnofsky unter den Patienten mit Therapieerfolg.......................34

Tabelle 17: Häufigkeit von Vorerkrankungen unter Patienten mit Therapieerfolg ..................35

Tabelle 18: Häufigkeit von Reoperationen nach initialer Minibohrlochtrepanation.................36

Tabelle 19: Altersverteilung unter den Patienten mit Reoperation ..............................................36

Tabelle 20: Pearson-Korrelation zwischen dem Patientenalter und der Anzahl der

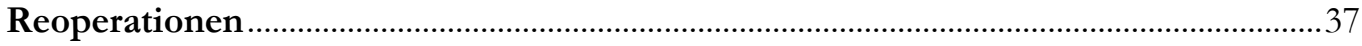

Tabelle 21: Leukozyten-, Thrombozyten-, Hämoglobinwerte der Patienten mit Reoperation.

Tabelle 22: Gerinnungswerte der Patienten mit Reoperation........................................................ 38

Tabelle 23: Häufigkeit von Antikoagulanzien unter Patienten mit Reoperation ......................39

Tabelle 24: Häufigkeit eines präoperativen Traumas bei Patienten mit Reoperation.............40

Tabelle 25: Einteilung nach Karnofsky bei Patienten mit Reoperation ....................................... 40

Tabelle 26: Häufigkeit von Vorerkrankungen bei Patienten mit Reoperation.............................41

Tabelle 27: Häufigkeit des Einsatzes invasiverer Operationsmethoden.......................................42



Tabelle 29: Altersverteilung unter den Patienten mit invasiverer Operationsmethode ...........43

Tabelle 30: Leukozyten-, Thrombozyten-, Hämoglobinwerte der Patienten mit invasiverer Operationsmethode.

Tabelle 31: Gerinnungswerte von Patienten mit invasiverer Operationsmethode .....................44

Tabelle 32: Häufigkeit von Antikoagulanzien unter den Patienten mit invasiverer

Operationsmethode.

Tabelle 33: Häufigkeit von präoperativem Trauma bei Patienten mit invasiverer Operationsmethode.

Tabelle 34: Einteilung nach Karnofsky-Index unter Patienten mit invasiverer Operationsmethode.

Tabelle 35: Häufigkeit der Vorerkrankungen unter den Patienten mit invasiverer Operationsmethode. 


\section{Abkürzungsverzeichnis}

$\begin{array}{ll}\text { ASS } & \text { Acetylsalicylsäure } \\ \text { cCT } & \text { kraniale Computertomographie } \\ \text { cSDH } & \text { chronisches Subduralhämatom } \\ \text { CT } & \text { Computertomographie } \\ \text { ICP } & \text { (intracranial pressure) intrakranieller Druck } \\ \text { INR } & \text { International normalized ratio } \\ \text { K } & \text { erweiterte Bohrlochtrepanation } \\ \text { K + D } & \text { erweiterte Bohrlochtrepanation mit Drainage } \\ \text { KHK } & \text { Koronare Herzkrankheit } \\ \text { M } & \text { Minibohrlochtrepanation } \\ \text { MRT } & \text { Magnetresonanztomographie } \\ \text { NMH } & \text { Niedermolekulares Heparin } \\ \text { OP } & \text { Operation } \\ \text { PTT } & \text { partielle Thromboplastinzeit } \\ \text { SDH } & \text { Subduralhämatom } \\ \text { TAA } & \text { Tachyarrythmia absoluta }\end{array}$




\section{$1 \quad$ Einleitung}

Das chronische Subduralhämatom $(\mathrm{cSDH})$ ist ein viele Jahrhunderte bekanntes Krankheitsbild, welches vor allem ältere Patienten betrifft.

Im 17. Jahrhundert wurde der erste Fall eines cSDH durch Johann Jacob Wepfer in seinem Werk Anatomicae ex Cadaveribus eorum quos sustulit Apoplexia explizit beschrieben.

Neuste Untersuchungen an den Gebeinen des Komponisten Wolfgang Amadeus Mozart (1756-1791) geben Indizien, dass es sich bei der Erkrankung, an der er mit nur 35 Jahren verstorben ist, um ein cSDH gehandelt haben könnte (Drake 1993).

Im Laufe des 19. Jahrhunderts beschäftigte sich die Wissenschaft intensiver mit dem Krankheitsbild. Als Ursache wurden Entzündungen oder Traumata vermutet. Erst 1857 veröffentlichte Virchow die erste wissenschaftliche Untersuchung zu cSDH. Er beschrieb eine entzündliche Ursache. Aufgrund seiner expliziten Ausführung wurde seine Erklärung von der Wissenschaft lange Zeit akzeptiert. Trotter stellte Virchows Theorie 1914 erstmalig in Frage. In dem Roman „Pierrette“ von Honoré de Balzac (1799-1850) wird schon sehr früh ein chronisches Subduralhämatom beschrieben, dem ein Trauma zugrunde lag (van den Doel 1986).

Die meisten chirurgischen Verfahren basieren bis heute auf der sog. Trepanation des Schädels (griech. Trypanon, lat. Trepanum = der Bohrer). Chronische Subduralhämatome stellen heute die häufigsten intrakraniellen Blutungen dar.

Trotz mittlerweile hinlänglich bekannter Pathogenese konnte bisher keine Einigkeit über die beste Therapie erreicht werden. Einige Aspekte des besten klinischen Managements werden noch kontrovers diskutiert (Ducruet et al. 2012).

Das Verständnis für Therapieversager, Risikofaktoren und Komplikationen bedarf weiterer Forschung. Es konnte nicht abschließend geklärt werden, welches Operationsverfahren sich patientenbezogen als geeignet erweist. Das Therapiespektrum reicht von Minibohrlochtrepanationen über erweiterte Bohrlöcher bis hin zu Kraniotomien. Bei der Minibohrlochtrepanation stellt sich insbesondere die Frage nach der Effektivität, respektive der Häufigkeit der Therapieversager. Bei einem Therapieversagen wird diskutiert, ob nicht generell eine zu invasive Therapie, also eine Übertherapie, zugrunde liegt.

Die vorliegende Arbeit soll erörtern, ob es präoperativ Indizien für Erfolg oder Misserfolg von Minibohrlochtrepanationen gibt, um das minimal-invasive Verfahren gezielter einzusetzen. 


\subsection{Inzidenz}

Die Zahl der cSDH ist ansteigend. Dieser Zusammenhang ist im steigenden Altersmittel der Bevölkerung und in der Altersabhängigkeit des Krankheitsbildes zu sehen. Die Inzidenz bei Patienten unter 65 Lebensjahren beträgt 3,4 pro 100.000 Einwohner, bei Patienten älter als 65 Jahre zwischen 8 und 58 pro 100.000 Einwohner. Die Angaben zur Altersabhängigkeit sind zunehmend relevant. Im Jahre 2003 betrug die Zahl der Menschen älter als 65 Jahre 12\% der Weltbevölkerung. Die Tendenz ist steigend (Ducruet et al. 2012).

In den USA wird eine Verdoppelung der Inzidenz von cSDH in den nächsten 25 Jahren erwartet. Weltweit ist dieser Trend annähernd gleich (Ducruet et al. 2012).

\section{2 Ätiologie}

Entgegen der Annahme von Virchow (1857) handelt es sich bei dem cSDH nicht um eine aseptische Entzündung (Pachymeningitis haemorrhagica interna). Häufig liegen traumatische Ereignisse vor dem Auftreten der ersten Symptome eines cSDH vor. Nakaguchi et al. (2000) geben an, dass $60-80 \%$ der cSDH traumatisch bedingt sind. Oftmals handelt es sich um ein Bagatelltrauma, an das sich die Patienten nicht erinnern. Es sind jedoch auch Fälle beschrieben, in denen ein cSDH ohne vorangegangene Unfälle aufgetreten ist.

Andere seltene Ursachen für eine subdurale Blutung können arteriovenöse Malformationen, Infektionen oder Tumorerkrankungen wie eine meningeale Karzimatose sein.

\section{Ätiologischer Einfluss von Begleiterkrankungen}

Vorzugsweise finden sich cSDH bei älteren Menschen, Alkoholikern, dialysepflichtigen Nierenpatienten oder bei Patienten mit hämatologischen Erkrankungen und Koagulopathien.

Ebenso besteht bei Diabetes mellitus und arteriellem Hypertonus der Verdacht, dass sie als prädisponierende Faktoren für cSDH wirken. Eine Ursache hierfür könnte in der gefäßschädigenden Komponente der Erkrankungen liegen.

Steimle et al. (1990) beispielsweise eruierten 26,5\% Hypertoniker in ihrem Kollektiv von Patienten mit cSDH.

Chronischer Alkoholmissbrauch führt zu einer Störung der Koagulation, wodurch das Risiko eines cSDH erhöht werden kann (Chen und Levy 2000). Es kommt zu einer Schädigung der Hepatozyten und damit zu einer gestörten Bildung der Vitamin-K-abhängigen Gerinnungsfaktoren. Ebenso kann bei chronischem Alkoholkonsum eine Hirnatrophie eintreten, die das Risiko weiter steigert (Markwalder et al. 1981). 
Ätiologischer Einfluss von Antikoagulanzien

Blutgerinnungsbeeinflussende Medikamente haben ein weites Einsatzspektrum.

Antikoagulierte Patienten haben ein erhöhtes Risiko, ein cSDH zu entwickeln. Der exakte Pathomechanismus konnte bisher nicht vollständig erklärt werden, aber es wird davon ausgegangen, dass die Medikation ein Stoppen von Mikroblutungen beeinträchtigt (Ducruet et al. 2012).

Die Inzidenz der cSDH hat signifikant zugenommen, seit Medikamente zur Antikoagulation vermehrt Einsatz finden (Ducruet et al. 2012).

Das Risiko für die Entstehung von cSDH ist bei Antikoagulanzien bis zu 42,5fach erhöht (Rust et al. 2006). In dieser Untersuchung nahmen $42 \%$ der Patienten mit vorangegangenem Trauma Gerinnungshemmer, aber 63\% der Patienten ohne erinnerliches Trauma. Es ist daher davon auszugehen, dass auch ohne Trauma eine medikamentöse Beeinflussung der Hämostase die Entwicklung eines cSDH fördern kann.

Patienten mit oral eingenommenem Warfarin haben nach Ansell et al. (2004) das höchste Risiko, ein cSDH zu entwickeln. Torihashi et al. (2008) stellten zudem fest, dass das Intervall zwischen Trauma und Erstoperation unter Antikoagulation kürzer war.

Es besteht allgemein Einigkeit darüber, dass Patienten mit symptomatischem cSDH unter Antikoagulation schnellstmöglich eine Normalisierung der Gerinnung benötigen, um eine Expansion der Blutung zu unterbinden und neurochirurgische Maßnahmen zu ermöglichen (Hanley 2004).

\section{Ätiologischer Einfluss von Hirnatrophie}

Rarefizierende Hirnveränderungen scheinen prädisponierend für die Entwicklung eines cSDH zu wirken.

Der signifikante Anstieg der Größe des Liquorraums ist Ausdruck eines physiologischen Alterungsprozesses. Ein zunehmendes Platzangebot kann eine Einblutung begünstigen (Meese et al. 1980).

Nach Wecht (2000) werden durch die Atrophie die Venen, die von der Hirnoberfläche zur Dura ziehen, unter Spannung gesetzt, wodurch sich das Risiko einer Ruptur vergrößert. 


\subsection{Pathogenese}

Aus pathophysiologischer Sicht wird bei der Entstehung eines cSDH von einem Einriss der Brückenvenen ausgegangen (Chen und Levy 2000). Ursächlich ist, wie erwähnt, oft ein Schädel-Hirn-Trauma, wobei Beschleunigungskräfte auf das Hirnparenchym und die Gefäße wirken und zu einer Einblutung in den subduralen Raum führen (Ducruet et al. 2012).

Ein niedriger intrakranieller Druck (ICP) beeinflusst, unabhängig von der Ursache, die Entstehung eines cSDH. Die Brückenvenen sind durch den niedrigen ICP prall gefüllt und anfälliger für Verletzungen (Piotrowski und Krombholz-Reindl 1996).

Bei gesunden Individuen lässt sich kein Subduralraum finden, da Dura mater und Arachnoidea direkt aufeinander liegen (Ducruet et al. 2012). Physiologischerweise wird der Subduralraum von einer dichten Zellschicht, dem Neurothel, ausgefüllt (Becker et al. 1988). Durch die Einblutung zwischen Dura mater und Arachnoidea entsteht die subdurale Kavität. Zunächst entwickelt sich das cSDH als eine kleine Blutung, um die sich nach etwa 14 Tagen eine vaskularisierte Membran bildet.

Diese Kapsel aus Granulationsgewebe ist eine Reaktion der Dura mater auf Blut, Fibrin oder Fibrinspaltprodukte (Ito et al. 1976, Markwalder et al. 1981). Die Kapselmembran wird auch Pseudomembran genannt (Piotrowski und Krombholz-Reindl 1996).

Ducruet et al. (2012) beschreiben, dass sich aus dem traumatisch bedingten, vorerst akuten SDH durch Organisation, enzymatische Fibrinolyse und Verflüssigung des subduralen Blutgerinnsels eine Entzündungsreaktion entwickelt. Die Kollagensynthese und Ausbreitung von Fibroblasten über die innere Oberfläche der Dura mater führt zu einer dicken äußeren Hämatommembran.

Durch langsame Größenzunahme und kontinuierliche Dehnung des Subduralraumes wird eine weitere Einblutung bedingt. Durch die Tendenz zur Vergrößerung treten Symptome erst ab einem kritischen Maß auf.

\subsection{Klinische Symptomatik}

Aufgrund der großen Symptomvielfalt ist es schwierig, ein cSDH klinisch zu diagnostizieren.

Die klinische Symptomatik reicht von Kopfschmerzen über Schwindel, Druck- und Klopfdolenz des betroffenen Areals, hirnorganische Verlangsamung, Verlust der Konzentrationsfähigkeit bis zu Orientierungslosigkeit und Psychosen. Oft zeigen sich auch latente oder manifeste Paresen, Sensibilitätsstörungen, epileptische Anfälle, Reflexdifferenzen oder Sprachstörungen. 
Da es sich in vielen Fällen um ältere Patienten handelt, kann eine Abgrenzung zur Demenz schwerfallen.

CSDH in der hinteren Schädelgrube manifestieren sich mit Abduzensparesen, Nystagmus und dyskonjugierter Bulbusstellung.

Es kann bei kritischem Ausmaß der Raumforderung auch bei chronischen Subduralhämatomen zu rascher Dekompensation mit zerebraler Einklemmung kommen.

\subsection{Diagnostik}

Die wichtigste diagnostische Maßnahme ist die kraniale Computertomographie (cCT). Die Computertomographie (CT) zeichnet sich durch eine hohe Sensitivität in der Diagnostik intrakranieller Prozesse aus (Kostanian et al. 2000).

Subduralhämatome stellen sich sichelförmig, konkav zum Hirngewebe dar und verdrängen das Hirnparenchym vom Schädel.

Je nach Alter des cSDH lassen sich in der cCT isodense, hypodense und hyperdense Areale unterscheiden. Das cSDH zeigt anfänglich ein hyperdenses Bild. Im Verlauf kommt es zu einer kontinuierlichen Dichteabnahme. Bilateral lokalisierte isodense Hämatome können in der cCT übersehen werden (Becker et al. 1988).

Abbildung 1 zeigt beispielhaft ein cCT eines beideitiges cSDH.

Aufgrund der schlechteren Verfügbarkeit und höheren Kosten der Magnetresonanztomographie (MRT) nimmt diese eine geringere Bedeutung in der Diagnostik ein.

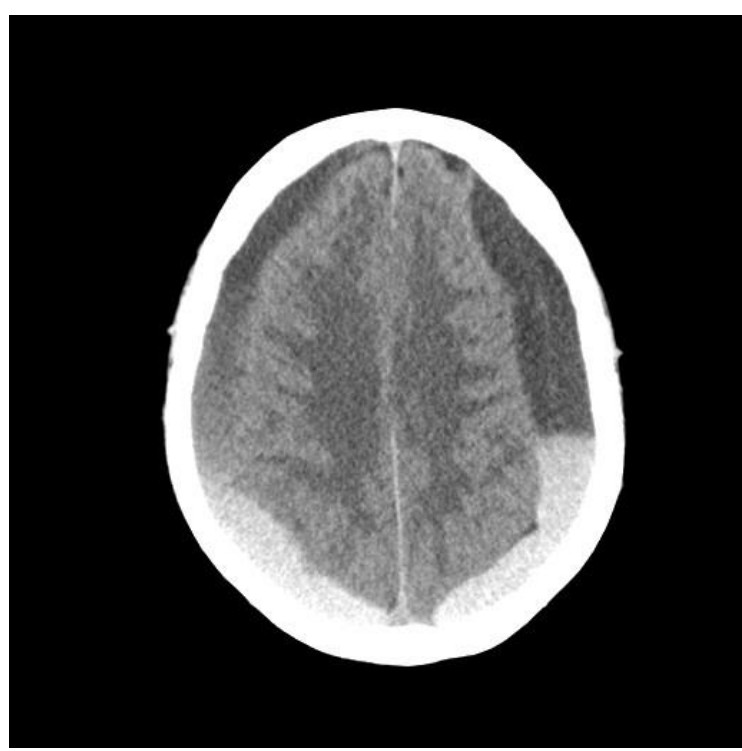

Abbildung 1: Computertomografische Darstellung eines beidseitigen cSDH 


\subsection{Therapieoptionen und Erfolgsraten}

CSDH gehören zu den häufigsten Ursachen für neurochirurgische Eingriffe (Rohde et al. 2002). Obwohl es bereits Untersuchungen zu erfolgreich durchgeführten nicht-chirurgischen Behandlungsmethoden gibt, ist man sich heute einig, dass die chirurgische Intervention die beste Therapie von cSDH darstellt.

Die Entscheidung zur konservativen oder chirurgischen Therapie wird anhand des Hämatomvolumens und der Symptomatik getroffen. Eine rein konservative Therapie ist denkbar, wenn das Hämatom unter $10 \mathrm{~mm}$ Dicke aufweist, bei Patienten mit nur milden oder keinen Beschwerden oder bei internistischen Hochrisikopatienten. In der Regel kommen dann zunächst Kortikosteroide zur Anwendung, die eine entzündungshemmende Wirkung auf die Neomembran haben. Eine Alternative bietet das Antifibrinolytikum Tranexamsäure, welches eine Verflüssigung des Hämatoms verhindern soll.

Spätestens bei Verschlechterung der Symptome bzw. erstmaligem Auftreten von Symptomen ist ein chirurgisches Vorgehen angezeigt und der nötige Umfang des chirurgischen Eingriffs zu wählen. Es bleibt zu klären, ob präoperative Parameter auf einen besseren Erfolg des einen oder des anderen operativen Verfahrens hinweisen können. Die optimale chirurgische Therapie bleibt daher weiterhin ein Diskussionsthema (Rohde et al. 2002).

\subsubsection{Operationsverfahren}

Die heute verfügbaren chirurgischen Methoden reichen von minimal-invasiven Minibohrlochtrepanationen über erweiterte Bohrlochtrepanationen mit oder ohne Einlage einer subduralen Drainage bis hin zu Kraniotomien (Ducruet et al. 2012).

\section{Minibohrlochtrepanation}

Unter Minibohrlochtrepanationen versteht man kleinste Eröffnungen des Schädels von 3-4 mm Durchmesser. In den meisten Fällen wird keine Drainage eingelegt.

Minibohrlochtrepanationen gelten als minimal-invasive Therapieoption für cSDH. Gerade in Hinblick auf die Behandlung von Hochrisikopatienten und um Übertherapien im Sinne von zu hoher Invasivität zu vermeiden, wurde die Minibohrlochtrepanation als Behandlungsalternative zur erweiterten Bohrlochtrepanation entwickelt und rückt mehr in den Fokus. Minibohrlochtrepanationen können am Bett des Patienten oder im OP durchgeführt werden. Abbildung 2 zeigt ein gängiges Instrumentarium für die Minibohrlochtrepanation.

Die Abbildungen 3-7 stellen das Verfahren der Minibohrlochtrepanation schrittweise dar. 


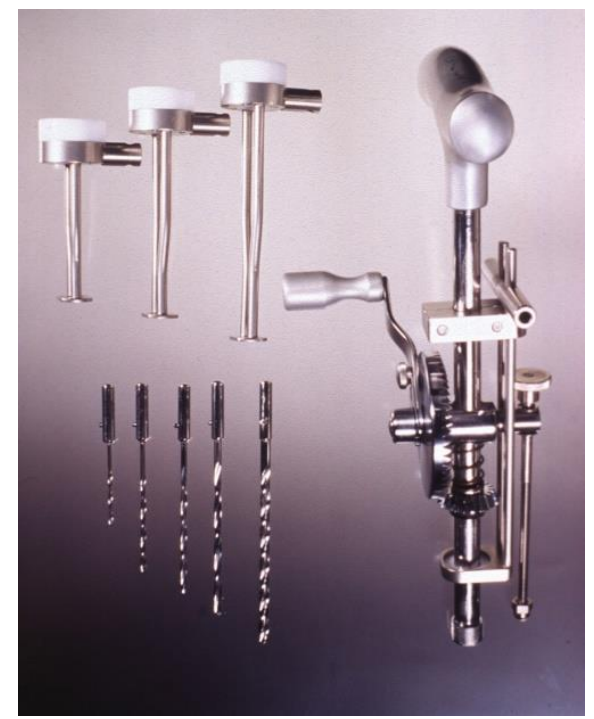

Abbildung 2: Instrumentarium für die Minibohrlochtrepanation (Drillbohrertrepanation)

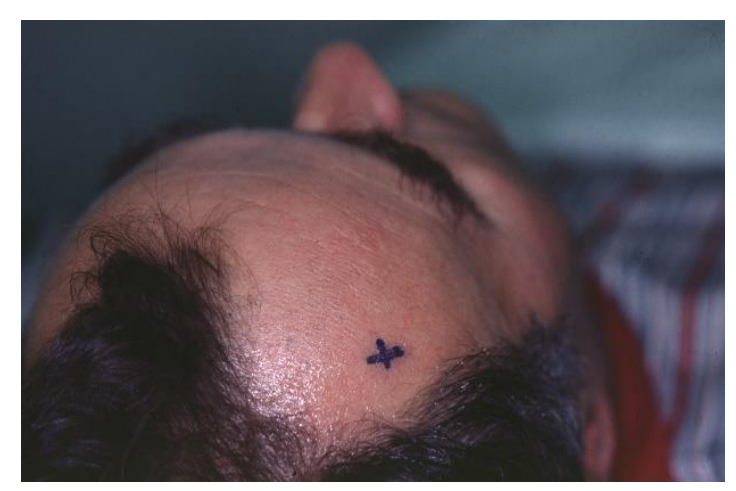

Abbildung 3: Minibohrlochtrepanation, Markierung der Trepanationsstelle



Abbildung 4: Minibohrlochtrepanation, Lokalanästhesie 


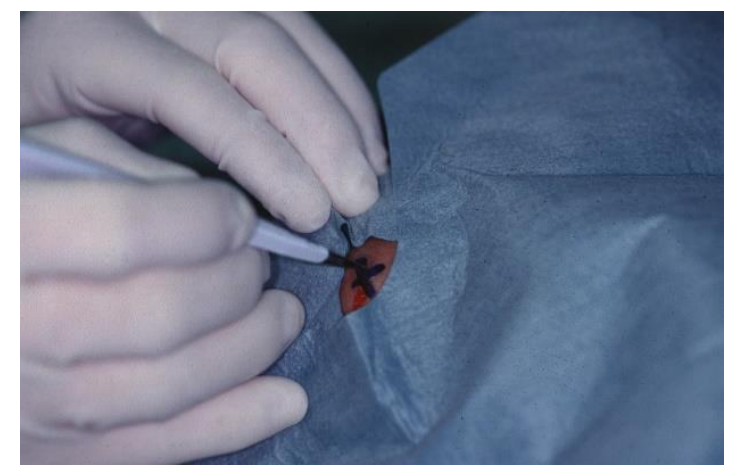

Abbildung 5: Minibohrlochtrepanation, Stichinzision

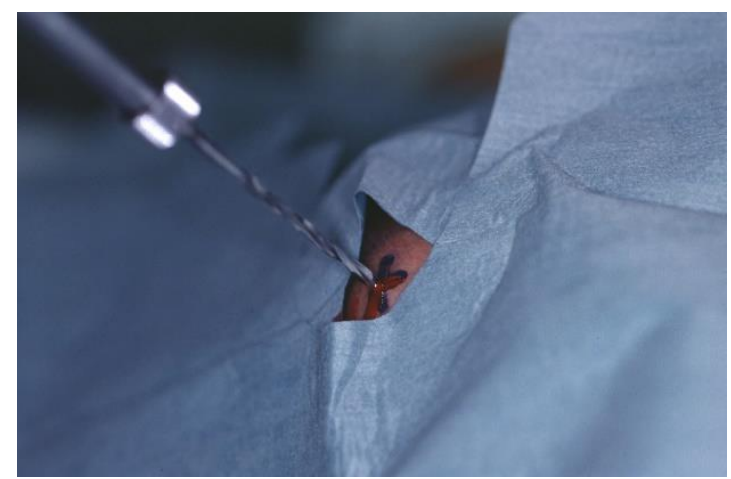

Abbildung 6: Minibohrlochtrepanation, Bohrung

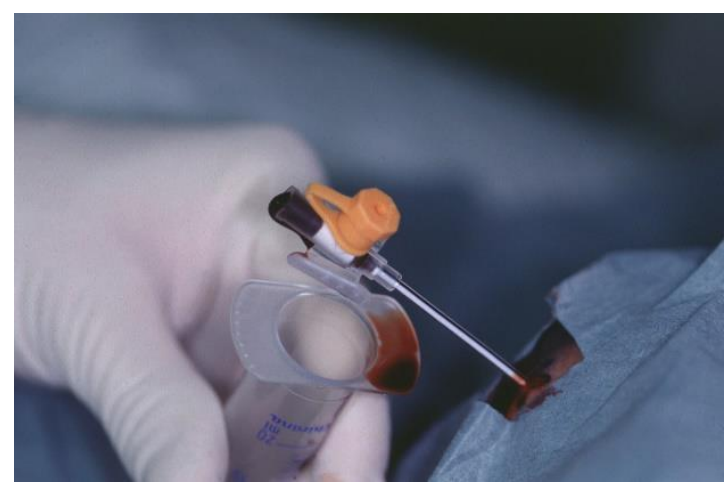

Abbildung 7: Minibohrlochtrepanation, Hämatomdrainage

Erweiterte Bohrlochtrepanation mit oder ohne Drainage

Die erweiterte Bohrlochtrepanation mit Anlage einer subduralen Drainage findet heute am häufigsten Verwendung in der Therapie der cSDH (Ducruet et al. 2012, Rohde et al. 2002, Reinges et al. 2000). In dem Review von Ducruet et al. (2012) wird zusammengefasst, dass 85\% der Befragten die erweiterte Bohrlochtrepanation der Kraniotomie und der Minibohrlochtrepanation als Initialtherapie vorziehen (Cenic et al. 2005). 
Hierbei erfolgt in der Regel eine Eröffnung des Schädels unter 30 mm (Weigel et al. 2003), wobei viele verschiedene Variationen dieses Operationsverfahrens bestehen (Ducruet et al. 2012).

Je nach Verfahren kommen ein oder zwei Bohrlöcher mit oder ohne Anlage einer subduralen Drainage zur Anwendung. Heute kann man eine erweiterte Bohrlochtrepanation in Intubationsnarkose oder Lokalanästhesie durchführen (Steimle et al. 1990).

Es zeichnet sich jedoch ein zunehmendes Interesse für minimal-invasive Behandlungsmethoden ab, da die erweiterte Bohrlochtrepanation aufgrund ihrer höheren Invasivität etwas höhere Operationsrisiken birgt.

\section{$\underline{\text { Kraniotomie }}$}

Schon frühzeitig wurde die Kraniotomie bei der Behandlung von cSDH beschrieben (Putnam und Cushing 1925). Diese Methode stellt die invasivste Operationstechnik mit den größten Operationsrisiken dar und besteht aus Entfernung eines Knochenstücks des Schädels über $30 \mathrm{~mm}$, welches nach Hämatomentfernung reimplantiert wird (Weigel et al. 2003).

Mit der gezielten präoperativen Diagnostik hat die Notwendigkeit einer Kraniotomie deutlich abgenommen, weswegen dieses Verfahren heute kaum noch zur Anwendung kommt. Sie sollte aufgrund der erhöhten Risiken nur im Falle von Hämatomen mehrerer Blutungslokalisationen durchgeführt werden (White et al. 2010) oder bleibt eine Option zur Entfernung von organisierten und kalzifizierten Hämatomen und cSDH mit mehreren Membranen (Ducruet et al. 2012).

\section{Seltene Operationsalternativen}

Eine interessante Methode zur Drainage von cSDH wurde von Takeda et al. (2006) beschrieben. Es wurde eine Sauerstoffinjektion in die Hämatomhöhle durchgeführt. Im zweiten Schritt wurde das gleiche Volumen mit einer Spritze aspiriert und abhängig vom Hämatomvolumen eine Rotation des Patientenkopfes von der Seiten- zur Rückenlage durchgeführt. Durch die beschriebene Technik konnte bei gleichem Rezidivrisiko der postoperative Kopfschmerz verringert werden. 


\subsubsection{Erfolge in der Therapie von cSDH}

Erfolge der Minibohrlochtrepanation

Reinges et al. (2000) führten an 118 Patienten Minibohrlochtrepanationen durch und konnten mit bis zu 5 Bohrungen $92 \%$ der Patienten mit unilateralem cSDH und mit bis zu 10 Bohrungen 95\% der Patienten mit bilateralem cSDH erfolgreich behandeln.

Die Indikation für eine Reoperation sahen die Autoren, wenn das reakkumulierte oder verbliebene Hämatomvolumen die Dicke des Schädelknochens hatte und der Patient klinische Symptome zeigte.

Die Entfernung des Hämatoms wurde durch spontane Drainage der Hämatomflüssigkeit unter Valsalva-Manöver und $30^{\circ}$-Trendelenburg-Lagerung erreicht. Die Autoren sehen in der Minibohrlochtrepanation die Möglichkeit, das Risiko von Infektionen und Verletzungen durch Verzicht auf eine subdurale Drainage zu reduzieren. Sie gehen davon aus, dass auch eine unvollständige Hämatomentlastung pathophysiologisch das Verhältnis von Reblutung und Reabsorption zugunsten der Reabsorption verschiebt und damit einen Selbstheilungsprozess initiiert, weshalb die komplette Hämatomentfernung nicht alleiniges Therapieziel darstellt.

Horn et al. (2006) verglichen die Effektivität der Minibohrlochtrepanation mit der erweiterten Bohrlochtrepanation. 55 Patienten mit cSDH erhielten eine Minibohrlochtrepanation, wohingegen 24 Patienten mit erweiterter Bohrlochtrepanation therapiert wurden. In dieser Studie war die Behandlung eines cSDH mit Minibohrlochtrepanation genauso effektiv wie jene mit erweiterten Bohrlochtrepanationen.

Yadav et al. (2013) modifizierten die Technik der klassischen Minibohrlochtrepanation. Ziel war es, das Komplikationsrisiko (Blutungen, Hirnverletzungen) bedingt durch die fehlende Sicht auf das OP-Gebiet noch weiter zu senken. Es wurden 50 Patienten operiert, die aufgrund ihres Allgemeinzustandes ein erhöhtes Anästhesierisiko aufwiesen. Die präferierte Bohrstelle lag im Bereich der größten frontalen und parietalen Krümmung. Es erfolgte eine Bohrung von $5 \mathrm{~mm}$ mit 90 Grad Angulation. Falls die größte Ausdehnung des Hämatoms nicht unter der präferierten Bohrstelle lag und die Bohrung auf flacherer Oberfläche durchgeführt werden musste, wurde der Bohrer 60 Grad anguliert. Diese Angulation gewährleistete einen einfachen und vergleichsweise geraden Zugang in die Hämatomkavität und verhinderte Hirnverletzungen bei Einführung einer Ernährungssonde für Kinder als Drainagesystem in die Hämatomkavität. Über dieses Drainagesystem konnte eine schrittweise Entfernung des Hämatoms durch gleichbleibenden extraduralen Unterdruck erreicht werden. In dieser Studie gab es keinen Fall einer Blutung oder einer Hirnverletzung. Yadav und Kollegen bezeichnen die modifizierte Minibohrlochtrepanation als gute, sichere, einfache und effektive Methode in der Behandlung von cSDH. 
Ducruet et al. (2012) betrachten die Minibohrlochtrepanation als am effektivsten bei nahezu vollständig verflüssigtem Blut und bezeichnen sie als eine gute Alternative für Patienten mit erhöhtem Operationsrisiko.

Für Patienten mit reduziertem Allgemeinzustand empfehlen zahlreiche Autoren die Trepanation am Krankenbett als erste, minimal-invasive Therapieoption.

Die Abbildungen 8-13 zeigen anhand von cCT-Aufnahmen beispielhaft den Verlauf eines einseitigen cSDH mit vollständiger Heilung durch Minibohrlochtrepanation.

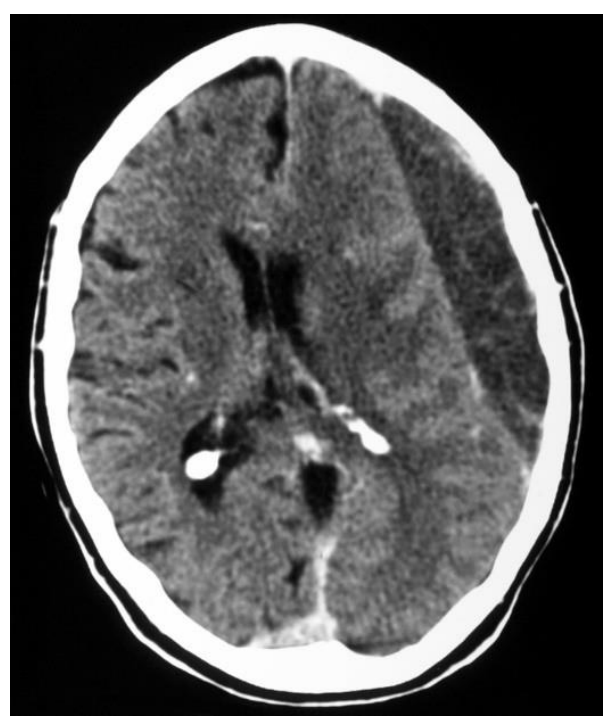

Abbildung 8: cSDH rechts, präoperativ

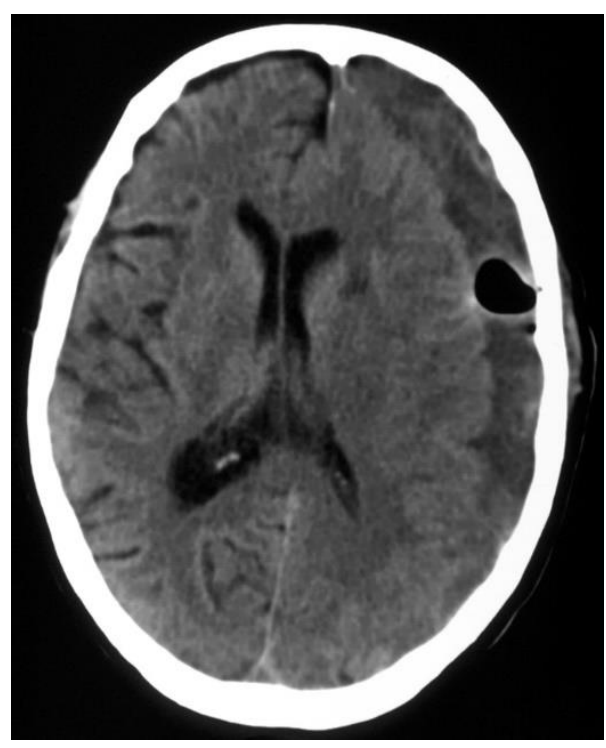

Abbildung 9: cSDH rechts, Erstkontrolle postoperativ 


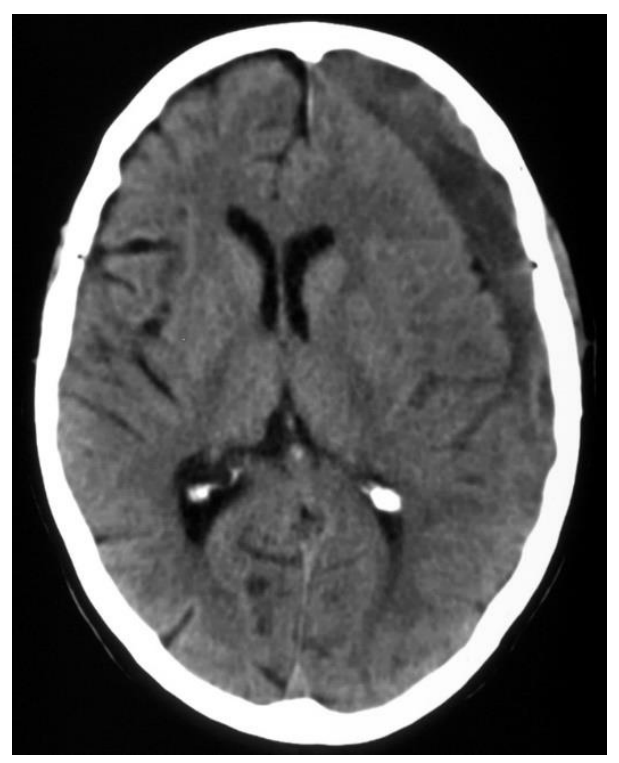

Abbildung 10: cSDH rechts, Verlaufskontrolle am 4. postoperativen Tag

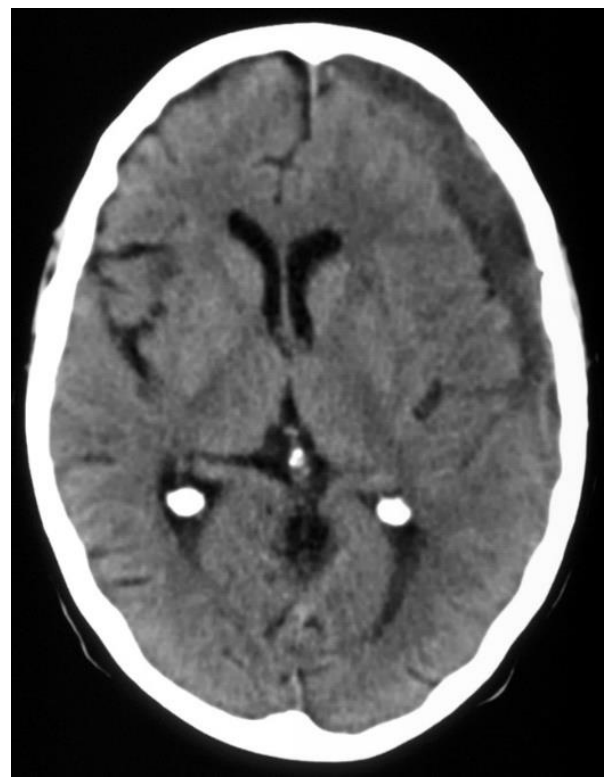

Abbildung 11: cSDH rechts, Verlaufskontrolle am 16. postoperativen Tag 


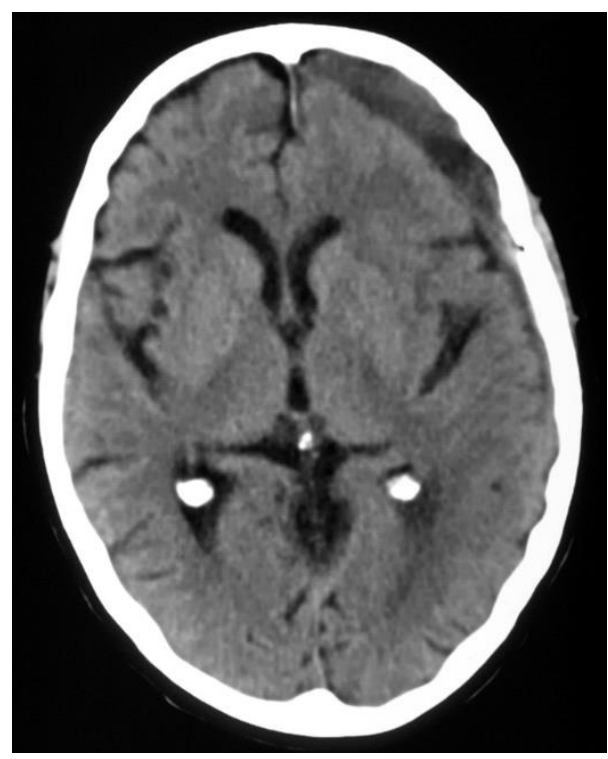

Abbildung 12: cSDH rechts, Verlaufskontrolle 6 Wochen postoperativ

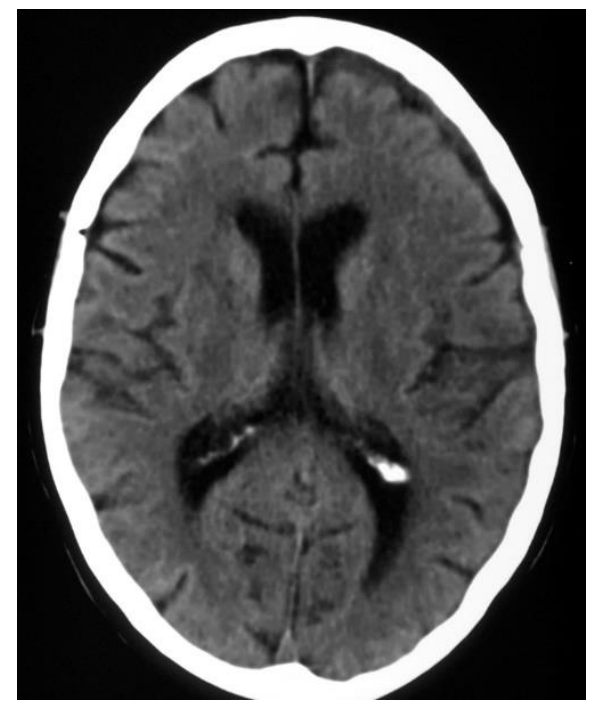

Abbildung 13: Verlaufskontrolle 14 Wochen postoperativ mit vollständiger Heilung des cSDH rechts

\section{Erfolge der erweiterten Bohrlochtrepanation}

In der Serie von Stanisic et al. (2005) wurden 99 Patienten mit 121 cSDH mit erweiterten Bohrlochtrepanationen therapiert. Bei den meisten Patienten kam ein Bohrloch zum Einsatz, bei zwei Patienten wurden zwei Bohrlöcher angewendet. Die Anlage einer subduralen Drainage erfolgte zu 67,8\%. 82,6\% der Patienten ließen sich durch den Initialeingriff heilen, 95,9\% durch zwei Eingriffe und bei 1,6\% war ein dritter Eingriff notwendig.

Sowohl Zhang et al. (2012) als auch Yadav et al. (2013) favorisieren die erweiterte Bohrlochtrepanation als die effizienteste und sicherste Methode in der Therapie eines cSDH. 
Erfolge der Operationsmethode nach Takeda et al. (2006)

Takeda et al. (2006) erreichten mit ihrer Operationsmethode der Sauerstoffinjektion in die Hämatomhöhle eine erfolgreiche Entfernung aller 77 behandelten Hämatome sowie eine zufriedenstellende neurologische Verbesserung von 70 Patienten.

\subsection{Rezidive und Komplikationen}

\subsubsection{Rezidive}

Die Rezidivraten von cSDH nach Therapie mit einem der aktuellen Operationsverfahren liegen laut Escosa Baé et al. (2011) zwischen 5-30\%.

In 0,35-33\% führen rezidivierende oder persistierende cSDH zu erneuten chirurgischen Interventionen (Drapkin 1991, Frati et al. 2004, Hamilton et al. 1993, Harders et al. 1981, Kotwica und Brzezinski 1991, Kuroki et al. 2001, Markwalder et al. 1981, Matsumoto et al. 1999, Murakami et al. 2002, Nagata et al. 1989, Nakaguchi et al. 2000, Nakaguchi et al. 2001, Nomura et al. 1994, Okada et al. 2002).

\section{$\underline{\text { Rezidive nach Minibohrlochtrepanation }}$}

In Bezugnahme auf vorhandene Untersuchungen von Camel und Grubb (1986), Rychlicki et al. (1991), Smely et al. (1997) und Tabaddor und Shulmon (1977) beschreiben die Autoren Escosa Baé et al. (2011), dass die Rezidivrate nach Minibohrlochtrepanation höher ist als nach erweiterten Bohrlochtrepanationen (33\% vs. 1-12\%).

Um zu untersuchen, ob der positive Einfluss von Drainagen auf die Rezidivrate von erweiterten Bohrlochtrepanationen auch auf die Rezidivrate von Minibohrlochtrepanationen zu übertragen ist und welche Prädiktoren es für ein Rezidiv nach Minibohrlochtrepanation gibt, untersuchten Escosa Baé et al. (2011) 312 Patienten.

Da es sich bei der Minibohrlochtrepanation um ein Verfahren handelt, welches das Hämatom nicht vollständig entleert, definierten die Autoren ein Wiederauftreten der Symptome und/oder einen Anstieg der Hämatomdicke im postoperativen CT innerhalb von 3 Monaten als Rezidiv. In diesen Fällen wurde eine Reoperation durchgeführt.

Im Vergleich dazu sahen Reinges et al. (2000) die Indikation für eine Reoperation, wenn das reakkumulierte oder verbliebene Hämatomvolumen die Dicke des Schädelknochens hatte und der Patient klinische Symptome zeigte.

Escosa Baé et al. (2011) kamen auf eine Rezidivrate von 12\% nach Minibohrlochtrepanation mit Drainage, welche gleich hoch ist zu der von ihnen für erweiterte Bohrlochtrepanationen 
definierten Rezidivrate. Auf vergleichbare Ergebnisse kamen auch Cenic et al. (2005), Ramachandran und Hegde (2007), Reinges et al. (2000), Rohde et al. (2002) sowie Weigel et al. (2003).

Zusammenfassend betrachtet betrug die Rezidivrate nach Minibohrlochtrepanation zwischen 9,8 und $33 \%$.

\section{$\underline{\text { Rezidive bei erweiterter Bohrlochtrepanation }}$}

Die Autoren Santarius et al. (2009) geben für das Verfahren der erweiterten Bohrlochtrepanation an, dass die Anlage einer subduralen Drainage das Rezidivrisiko nach erweiterten Bohrlöchern senkt. Sie arbeiteten einen signifikanten Nutzen der subduralen Drainage für die Rezidivrate, Mortalität und Abflussmenge nach Hämatomentlastung mit zwei Bohrlöchern heraus.

Sie untersuchten den Einfluss von Drainagen auf die Rezidivrate und das Outcome der Patienten anhand von 269 Fällen. Es ergab sich eine Rezidivrate von 9,3\% für Patienten mit Drainage und eine Rate von 24\% für Patienten ohne Drainage nach erweiterter Bohrlochtrepanation.

Diese Rezidivrate der erweiterter Bohrlochtrepanation mit Drainage wurde durch Stanisic et al. (2013) und Mori und Maeda (2001) bestätigt. Stanisic et al. (2013) errechneten eine Rezidivrate von $11,9 \%$, Mori und Maeda (2001) eine Rate von 9,8\%.

In der Untersuchung von Stanisic et al. aus dem Jahr 2005 ergab sich eine Rezidivrate nach Operation mit einem erweiterten Bohrloch von 14,9\%. Die Anlage einer Drainage erfolgte zu $67,8 \%$.

Tahsim-Oglou et al. (2012) wiederum kamen bei 247 Patienten nach zwei Bohrlöchern und Drainage auf eine Rezidivrate von 25,1\%.

In der Zusammenfassung beträgt die Rezidivrate nach erweitertem Bohrloch ohne Drainage zwischen 18 bis $24 \%$, mit Drainage zwischen 9,3 bis $25,1 \%$.

\section{Weitere Faktoren für Rezidive unabhängig vom Operationsverfahren}

Weiterhin relevant für die Rezidivneigung ist die Reexpansion des Gehirns nach operativer Entfernung der Blutung. Mori und Maeda (2001) zufolge ist bei Patienten mit Rezidiv die Reexpansion eine Woche postoperativ deutlich geringer als bei Patienten ohne Rezidiv. Patienten über 70 Jahre hatten eine deutlich geringere postoperative Reexpansion des Gehirns 
als Patienten unter 70 Jahre. Ein persistierender vergrößerter Subduralraum oder eine geringere Expansion haben einen geringeren tamponierenden Effekt, wodurch eine erneute Einblutung ermöglicht wird.

Ebenso beeinträchtigen bereits stattgefundene Schlaganfälle und eine subdurale Luftansammlung die zerebrale Reexpansion (Mori und Maeda 2001).

Da es durch Antikoagulanzien zu medikamentenassoziierten Reblutungen kommen kann, besteht ein Therapiekonflikt. Um weitere Einblutungen in die Hämatomkavität zu vermeiden, sollten ausgeprägte Koagulopathien bei Patienten mit cSDH rasch therapiert werden (Ducruet et al. 2012). Die Autoren beschreiben, dass subdurale Flüssigkeitsansammlungen und deren Resorption und Organisation durch Hämophilie, disseminierte intravasale Gerinnung und Thrombopathie beeinträchtigt werden können. Die Korrelation zwischen anamnestischen Faktoren, Antikoagulanzien und Rezidiven von cSDH ist Gegenstand vieler Untersuchungen. So beispielsweise konnten Mori und Maeda (2001) für antikoagulierte Patienten eine erhöhte Rezidivrate von 18,5\% feststellen, bei einer Rezidivrate von 9,8\% im Gesamtkollektiv. Tahsim-Oglou et al. (2012) untersuchten den Einfluss postoperativ verabreichter niedermolekularer Heparine $(\mathrm{NMH})$ auf Reblutungen von cSDH. Die Rezidivrate bei Patienten ohne postoperative Gabe von NMH war deutlich geringer als unter NMH bei einer durchschnittlichen Rezidivrate von $25,1 \%$.

Im Gegensatz dazu konnten Escosa Baé et al. (2011) keinen Zusammenhang zwischen antikoagulativer Medikation und der Rezidivrate nach Minibohrlochtrepanation mit Drainage ermitteln.

Ein Rezidiv eines cSDH kann ebenfalls durch anderweitig bedingte Nachblutungen ausgelöst werden. Die äußere Hämatommembran besteht aus einer Schicht eines dünnwandigen, sinusförmigen Gefäßsystems mit losen Zellverbindungen. Plötzliche Druckveränderungen innerhalb des Hämatoms können zu einem Einriss dieses fragilen neuen Gefäßsystems führen. Diese Einblutungen in die Hämatomkavität können zu einem erneuten Hämatom führen oder das verbliebene Volumen ansteigen lassen (Escosa Baé et al. 2011). Die Autoren beobachteten in 21\% der untersuchten Fälle eine solche akute Einblutung nach dem chirurgischen Eingriff.

\subsubsection{Komplikationen}

Neben Rezidiven können weitere postoperative Komplikationen in der Behandlung von cSDH auftreten.

Es können Infektionen, wie oberflächliche Wundinfektionen, subdurale Empyeme oder Abszesse, epidurale Abszesse, Meningitiden, Osteomyelitiden oder Hirnabszesse auftreten. 
Darüber hinaus kann es Luftansammlungen im Subduralraum geben. Meistens verursachen diese keine Symptome, können aber zu erhöhtem Hirndruck führen. Auch Hirnödeme sind möglich, welche durch das subdurale Hämatom initiiert worden sind, sich aber postoperativ vergrößern und zu zerebralen Einklemmungen führen können. Weitere postoperative Komplikationen können epidurale Nachblutungen, Hirninfarkte, intrazerebrale Blutungen, Krampfanfälle oder akute SDH durch Verletzung eines Gefäßes sein.

Ebenso können internistische Komplikationen wie Infektionen, Bronchitiden, Pneumonien, Lungenödeme, Herzrhythmusstörungen, Herzinsuffizienzen oder gastrointestinale Probleme auftreten. In der Publikation von Rohde et al. (2002) wird unterschieden zwischen chirurgischen und medizinischen Komplikationen nach erweiterter Bohrlochtrepanation mit Drainage. Die häufigsten chirurgischen Komplikationen waren mit 13,6\% fokale oder generalisierte Krampfanfälle. Zu je 2,1\% ist es zu einer intrazerebralen Blutung oder einem subduralen Empyem gekommen. Symptomatische epidurale Hämatome traten in 1,3\% auf, ein symptomatischer Pneumocephalus in 1,1\% und ein intrazerebraler Abszess in $0,3 \%$.

Unter den medizinischen Komplikationen während des stationären Aufenthaltes fand sich als häufigste Komplikation eine Pneumonie (7,7\%). Die medizinischen Komplikationen hatten einen wesentlichen Anteil an der Gesamtmortalitätsrate von 13,3\%.

Das Risiko eines Pneumozephalus, welcher zu erhöhtem Hirndruck führen kann, besteht bei allen Operationsmethoden. Mit der Minibohrlochtrepanation ist das Risiko geringer (Reinges et al. 2000).

\subsection{Prognose}

Die Prognose für Patienten mit cSDH ist heute in der Regel gut (El Khadi et al. 2000). Anfänglich war die Kraniotomie noch mit einer hohen Mortalitätsrate von bis zu 30\% verbunden (Becker et al. 1988). Diese konnte durch die erweiterte Bohrlochtrepanation gesenkt werden. Die Gesamtmortalitätsrate nach erweiterter Bohrlochtrepanation mit Drainage wird von Rohde et al. (2002) mit 13,3\% beschrieben.

Der nächste große Schritt wurde durch die Minibohrlochtrepanation (twist-drill-Methode) erreicht. Horn et al. (2006) geben nach Minibohrlochtrepanation eine Mortalität von lediglich $7 \%$ an.

In den Ergebnissen von Reinges et al. (2000) lag die Mortalitätsrate bei 1\%. Ein Patient verstarb während des stationären Aufenthaltes an einem Schlaganfall nach kardiogener Embolie, jedoch keiner der Patienten an den Folgen des cSDH und der Operation. 


\section{Fragestellung und Ziel der Studie}

Ziel dieser retrospektiven Analyse ist es, Parameter zu finden, die ein optimales Therapieansprechen oder Therapieversagen einer Minibohrlochtrepanation vorhersagen. In den letzten Jahren ist die Minibohrlochtrepanation als minimal-invasives Verfahren zunehmend in den Fokus gerückt, da gemäß Literatur das Operationsverfahren ein geringeres operatives Risiko in Hinblick auf Infektionen, Verletzungen und Blutungen hat.

Gerade in Hinblick auf die Behandlung von Hochrisikopatienten mit reduziertem Allgemeinzustand und aufgrund der gewonnenen Kenntnisse zu Komplikationen und Mortalitätsrate bei cSDH ist die Minibohrlochtrepanation von Interesse, um Übertherapien im Sinne von zu hoher Invasivität zu vermeiden.

Auch ist die Mortalitätsrate geringer als bei der erweiterten Bohrlochtrepanation ohne und mit Drainage, die vielerorts noch das Standardverfahren darstellt. Die Effektivität (und damit die Rezidivrate) der Minibohrlochtrepanation wird noch kritisch gesehen, auch wenn Horn et al. (2006) und Escosa Baé et al. (2011) eine ähnliche Effektivität beschreiben.

Vor diesem Hintergrund initiierten wir die vorgelegte retrospektive Analyse mit dem Ziel, Parameter zu identifizieren, die optimales Therapieansprechen oder Therapieversagen der Minibohrlochtrepanation vorhersagen.

In Anlehnung an die Untersuchung von Mori und Maeda (2001) wurden auch die Analyse von internistischen Vorerkrankungen und in Anlehnung an die Untersuchungen von König et al. (2003), Yasuda et al. (2003) und Oishi et al. (2001) die Analyse der Laborparameter aus Blutbild und Gerinnung als Einflussfaktoren auf den Therapieerfolg eingeschlossen. 


\section{$3 \quad$ Material und Methoden}

\subsection{Patientenkollektiv}

Das Patientenkollektiv besteht aus Daten von 267 Patienten mit chronischen Subduralhämatomen. Alle Fälle wurden zwischen 2007 und einschließlich 2011 in der Klinik für Neurochirurgie der Georg-August-Universität Göttingen behandelt.

Kinder und Säuglinge mit cSDH, akute und subakute Verlaufsform oder subdurale Hygrome wurden nicht eingeschlossen.

Die Identifikation der Patienten erfolgte anhand der Operationsdokumentation, weitere Informationen wurden den Arztbriefen entnommen.

Einige Patienten waren von einem beidseitigen cSDH betroffen. Diese Patienten werden als zwei separate Fälle gewertet.

\subsection{Datenerfassung}

Die OP-Dokumentation stellt die Grundlage der erhobenen Daten dar. Es wurden Name, Vorname, Geburtsdatum, Geschlecht des Patienten, Zeitpunkt der Erst-Operation sowie Datum, Anzahl und Technik aller Reoperationen erfasst.

Alle Patienten erhielten als initiale Operation eine Minibohrlochtrepanation.

In Anlehnung an die Methodik von Reinges et al. (2000) kamen in unserer Untersuchung Minibohrlochtrepanationen mit 3-4 mm Durchmesser zur Anwendung. Es wurde keine Drainage angelegt. Das Drainieren erfolgte ausschließlich intraoperativ über eine Venenverweilkanüle.

Die dokumentierten Operationsverfahren wurden unterschieden in Minibohrlochtrepanation $(M)$, erweiterte Bohrlochtrepanation ohne $(K)$ oder mit Drainage $(K+D)$. Die weiteren Informationen wurden den zugehörigen Arztbriefen und digitalisierten Datenblättern entnommen.

Bei allen Patienten erfolgte präoperativ bei der Erstoperation eine Erfassung der Leukozyten

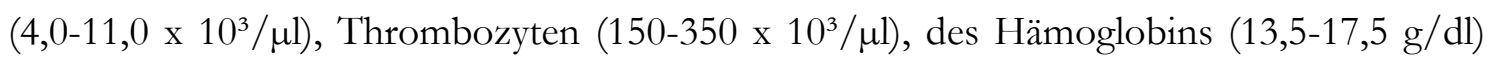
und der Gerinnungswerte Quick (70-130\%) und PTT (26-37 sec).

Aus dem jeweiligen Anamnesebogen wurden Informationen zur präoperativen Medikation gezogen und dokumentiert, ob Präparate mit Auswirkung auf die plasmatische Gerinnung und die Thrombozytenaggregation vorlagen. Die Wirkstoffe Phenprocoumon (Marcumar $\left.{ }^{\circledR}\right)$, Acetylsalicylsäure (ASS () ), Clopidogrel (Iscover $\left.{ }^{\circledR}\right)$ sowie niedermolekulare Heparine waren von Relevanz. 
Zugrunde liegende Erkrankungen wie Tachyarrythmia absoluta (TAA), koronare Herzkrankheit (KHK) oder ein erfolgter operativer Klappenersatz wurden dokumentiert.

Darüber hinaus wurden Angaben zu einem präoperativen Trauma, Hypertonie, Diabetes mellitus, symptomatische Epilepsie und einem postoperativen Empyem den Anamnesebögen und Arztbriefen entnommen.

Die Patienten wurden präoperativ anhand des Karnofsky-Index bewertet.

Alle Angaben, die bejaht oder verneint wurden, wurden zum Zwecke der erleichterten statistischen Auswertung für Ja mit 1, für Nein mit 0 verschlüsselt.

\subsection{Definition von Erfolg}

Patienten mit bis zu drei Minibohrlochtrepanationen werden als „Therapieerfolg“ gewertet. Patienten mit drei und mehr Minibohrlochtrepanationen oder einem nötigen Wechsel auf eine invasivere OP-Methode wurden als „Therapieversagen“ gewertet. 


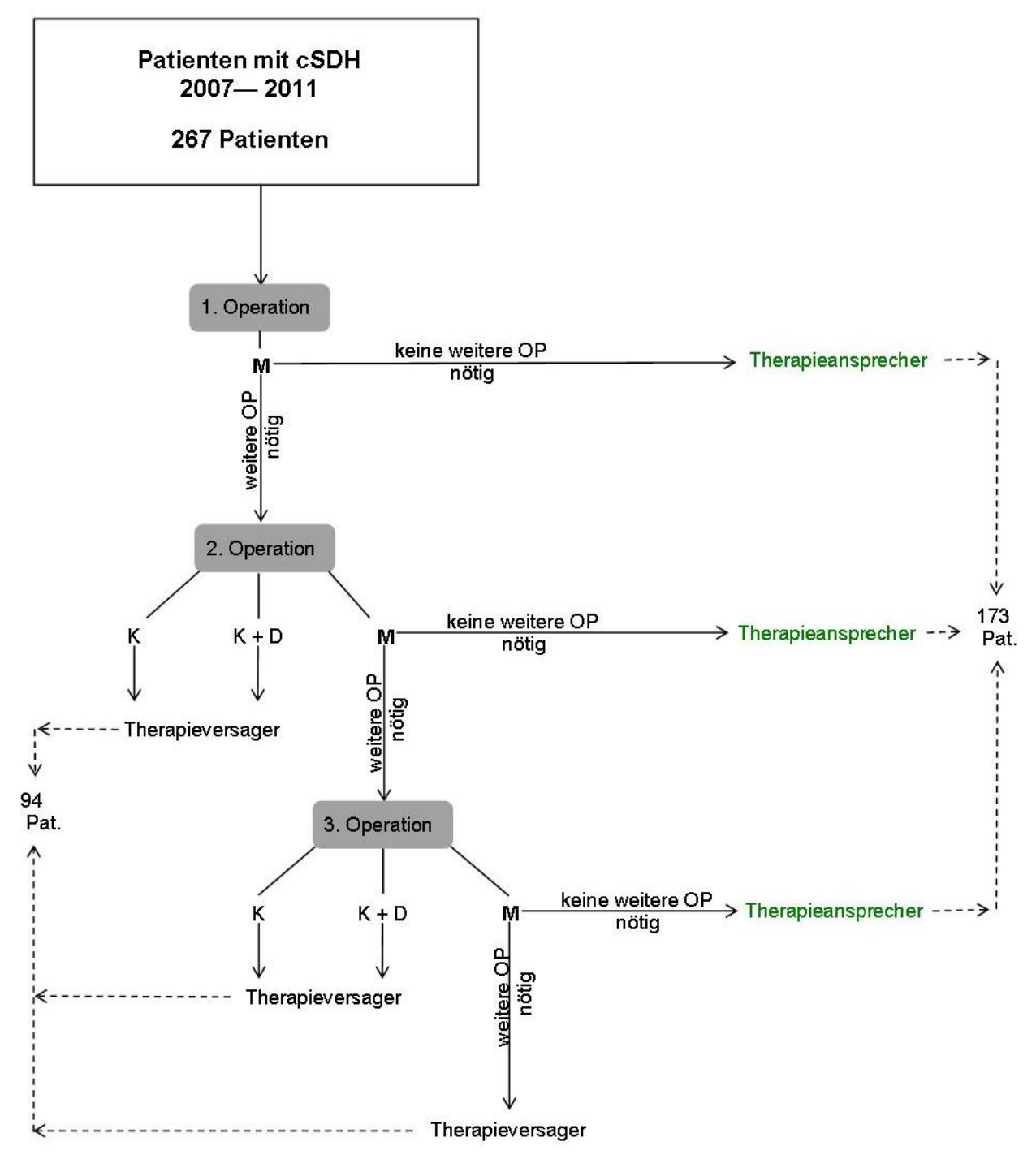

Abbildung 14: Übersichtsdiagramm zur Datenerhebung

$\mathbf{M}=$ Minibohrlochtrepanation, $\mathrm{K}=$ erweiterte Bohrlochtrepanation ohne Drainage, $\mathrm{K}+\mathrm{D}=$ erweiterte Bohrlochtrepanation mit Drainage

Abbildung 14 stellt die Methodik der Datenerfassung dar. Anhand der Erfolgsdefinition (siehe 3.3) ergeben sich Therapieerfolg und -versagen.

Es ist zu entnehmen, dass alle Patienten, die nicht diesen Kriterien entsprachen, als Therapieversager eingestuft wurden. 


\subsection{Einteilung der Patienten nach Karnofsky-Index}

Die Bewertung von Selbstversorgung, Selbstbestimmung und symptombezogenen Einschränkung der Patienten richtet sich nach der Einteilung von Karnofsky und Burchenal (1949).

Die Bewertung erfolgte vor der initialen Operation.

100\% keine Beschwerden, keine Zeichen der Krankheit

90\% fähig zu normaler Aktivität, keine oder geringe Symptome

80\% normale Aktivität mit Anstrengung möglich, deutliche Symptome

70\% Selbstversorgung, normale Aktivität oder Arbeit nicht möglich

60\% einige Hilfestellung nötig, selbstständig in den meisten Bereichen

50\% Hilfe und medizinische Versorgung wird oft in Anspruch genommen

40\% behindert, qualifizierte Hilfe nötig

$30 \%$ schwerbehindert, Hospitalisation erforderlich

20\% schwerkrank, intensive medizinische Maßnahmen erforderlich

10\% moribund, unaufhaltsamer körperlicher Verfall

$0 \% \quad$ Tod

Da Patienten ab einem Karnofsky-Index unter 50\% regelmäßig auf fremde Hilfe angewiesen sind, wurde diese Definition in der statistischen Auswertung für den Cutoff zugrunde gelegt.

\subsection{Statistische Methoden}

Hilfsmittel zur Erstellung der Tabelle nach Datenerhebung war das Programm Microsoft Excel.

Die genutzte Statistik-Software war SPSS Inc. Released 2009, PASW Statistics for Windows, Version 18.0 Chicago: SPSS Inc.

Zur Darstellung der absoluten sowie der prozentualen Anteile der jeweiligen Gruppen an der Gesamtpopulation der Studie wurden deskriptive statistische Methoden angewendet.

Multivariate Analysen zur Untersuchung mehrerer statistisch signifikanter Variablen waren bei den aktuellen Ergebnissen nicht nötig, da sie keine Hinweise auf Zusammenhangs- oder Abhängigkeitsstrukturen gegeben haben. 


\subsubsection{Chi-Quadrat-Test}

Der Chi-Quadrat-Test wurde zum Vergleich der Anteile der untersuchten Gruppe genutzt.

Es wird berechnet, ob unterschiedliche Merkmalsausprägungen mit ihren Häufigkeiten zufällig sind. Eine erwartete wird mit der tatsächlichen Häufigkeit verglichen. 5\% entsprechen dem Signifikanzniveau. Bei einem p-Wert kleiner als 0.05 wird davon ausgegangen, dass verschiedene Häufigkeitswahrscheinlichkeiten statistisch signifikant unterschiedlich sind.

\subsubsection{Streudiagramme / scatter plots}

Streudiagramme wurden erstellt, um kontinuierliche Variablen wie Laborwerte mit dem Alter und der Anzahl der Revisionsoperationen zu korrelieren. Es handelt sich um eine graphische Darstellung der beobachteten Wertepaare zweier statistischer Merkmale. Die Wertepaare wurden in ein kartesisches Koordinatensystem eingetragen, woraus eine Punktwolke entsteht. Es lassen sich daraus Informationen über die Abhängigkeitsstruktur der beiden Wertepaare ziehen.

Streudiagramme wurden zu den Wertepaaren Alter/Anzahl der Reoperationen, Leukozytenanzahl/Anzahl der Reoperationen, Thrombozytenzahl/Anzahl der Reoperationen, QuickWerte/Anzahl der Reoperationen, PTT-Werte/Anzahl der Reoperationen und Hämoglobinwerte/Anzahl der Reoperationen erstellt.

\subsubsection{Dichotomisierung}

Die Dichotomisierung beinhaltet die Zerlegung einer Gesamtheit in zwei Teilgesamtheiten anhand eines Merkmals, bei dem zwei Ausprägungen unterschieden werden.

Dichotomisiert wurden die Merkmale Leukozytenzahlen, Thrombozytenzahlen, Hämoglobinwerte, Quick und PTT. Anschließend wurde eine Boolesche Variable erstellt mit 0 oder 1 als zugewiesenem Wert. Die Toleranzgrenzen wurden folgendermaßen definiert:

\section{Leukozyten}

Referenzbereich 4,0-11,0 × $10^{3} / \mu \mathrm{l}$

$>11,0 \times 10^{3} / \mu 1=0$

$<11,0 \times 10^{3} / \mu l=1$

\section{Thrombozyten}

Referenzbereich $150-350 \times 10^{3} / \mu \mathrm{l}$

$<150 \times 10^{3} / \mu \mathrm{l}=0$

$>150 \times 10^{3} / \mu \mathrm{l}=1$ 
$\underline{\text { Hämoglobin }}$

Referenzbereich 13,5-17,5 g/dl

$<13,5 \mathrm{~g} / \mathrm{dl}=0$

$>13,5 \mathrm{~g} / \mathrm{dl}=1$

Quick

Referenzbereich 70-130\%

$<70 \%=0$

$>70 \%=1$

$\underline{\text { PTT }}$

Referenzbereich 26-37 sec

$>37 \mathrm{sec}=0$

$>37 \mathrm{sec}=1$ 


\section{$4 \quad$ Ergebnisse}

\subsection{Basisdaten}

Unter den 267 Patienten waren 188 (70,4\%) männlichen und 79 (29,6\%) weiblichen Geschlechts. Es gab 169 Patienten bis 80 Jahre (63,3\%) und 98 Patienten älter als 80 Jahre $(36,7 \%)$ (Tabelle 1).

Tabelle 1: Geschlechter- und Altersverteilung innerhalb des Patientenkollektivs

\begin{tabular}{|rr|r|r|}
\hline & Anzahl (n) & Anteil (\%) \\
\hline Geschlecht & M & 188 & 70,4 \\
& W & 79 & 29,6 \\
\hline älter als 80 Jahre nein & 169 & 63,3 \\
& ja & 98 & 36,7 \\
\hline
\end{tabular}

Die Verteilung der Leukozyten-, Thrombozyten- und Hämoglobinwerte ist Tabelle 2 zu entnehmen. Bei 239 Patienten (89,5\%) lagen die Leukozytenwerte unter 11 x 103/ $\mu$ l, 128 Patienten $(10,5 \%)$ hatten eine Leukozytose.

Die Thrombozytenwerte waren bei 242 Patienten $(90,6 \%)$ im Normbereich $\left(>150 \times 10^{3} / \mu \mathrm{l}\right)$. Insgesamt 25 Patienten $(9,4 \%)$ wiesen erniedrigte Werte auf.

Die Hämoglobinwerte waren bei 109 Patienten (40,8\%) im Normbereich (> 13,5 g/dl). Bei 158 Patienten (59,2\%) waren die Hämoglobinwerte erniedrigt.

Tabelle 2: Verteilung der Leukozyten-, Thrombozyten- und Hämoglobinwerte

\begin{tabular}{|lr|r|r|}
\hline & & Anzahl (n) & Anteil (\%) \\
\hline Leukozyten $<\mathbf{1 1}\left(\mathbf{1 0 ^ { 3 }} / \boldsymbol{\mu l}\right)$ & nein & 128 & 10,5 \\
& ja & 239 & $\mathbf{8 9 , 5}$ \\
\hline Thrombozyten $>\mathbf{1 5 0}\left(\mathbf{1 0}^{\mathbf{3}} / \boldsymbol{\mu l}\right)$ & nein & 25 & 9,4 \\
& ja & 242 & 90,6 \\
\hline Hämoglobin $>\mathbf{1 3 , 5} \mathbf{g} / \mathbf{d l}$ & nein & 158 & 59,2 \\
& ja & 109 & 40,8 \\
\hline
\end{tabular}


Die Gerinnungswerte Quick und PTT zeigt Tabelle 3. Ein Quick-Wert über 70\% im Normbereich lag bei 206 Patienten (77,2\%) vor. Einundsechzig Patienten (22,8\%) hatten einen zu geringen Quick-Wert.

Die PTT war bei 241 Patienten (90,3\%) regelrecht im Referenzbereich von 26 bis 37 Sekunden. Bei 26 Patienten (9,7\%) lag der Wert oberhalb dieses Bereiches.

Tabelle 3: Verteilung der Gerinnungswerte

\begin{tabular}{|rr|r|r|}
\hline & Anzahl (n) & \multicolumn{2}{|c|}{ Anteil in \% } \\
\hline Quick $>$ 70\% & nein & 61 & 22,8 \\
& ja & 206 & 77,2 \\
\hline PTT $<37$ sec & nein & 26 & 9,7 \\
& ja & 241 & 90,3 \\
\hline
\end{tabular}

Die Ergebnisse zu präoperativen Antikoagulanzien sind Tabelle 4 zu entnehmen.

Einundsiebzig Patienten (26,7\%) standen unter Phenprocoumon-Medikation. Achtunddreizig Patienten (14,3\%) bekamen präoperativ Acetylsalicylsäure. Acetylsalicylsäure in Kombination mit Clopidogrel wurden bei acht Patienten (3,0\%) verabreicht. Eine Kombination aus Phenprocoumon und Acetylsalicylsäure erhielten vier Patienten (1,5\%). Therapeutisch verabreichtes niedermolekulares Heparin $(\mathrm{NMH})$ war bei 16 Patienten $(6 \%)$ zu eruieren. Prophylaktisch erhielt keiner der Patienten niedermolekulares Heparin. 
Tabelle 4: Verteilung der präoperativen Antikoagulanzien

\begin{tabular}{|c|c|c|}
\hline & Anzahl (n) & Anteil (\%) \\
\hline \multicolumn{3}{|l|}{ Phenprocoumon } \\
\hline nein & 195 & 73,3 \\
\hline ja & 71 & 26,7 \\
\hline \multicolumn{3}{|l|}{ Acetylsalicylsäure } \\
\hline & 228 & 85,7 \\
\hline ja & 38 & 14,3 \\
\hline \multicolumn{3}{|l|}{ Acetylsalicylsäure + } \\
\hline & 258 & 97,0 \\
\hline ja & 8 & 3,0 \\
\hline \multicolumn{3}{|l|}{ Phenprocoumon +} \\
\hline nein & 262 & 98,5 \\
\hline ja & 4 & 1,5 \\
\hline \multicolumn{3}{|l|}{$\begin{array}{r}\text { Niedermol. Heparin } \\
\text { therapeutisch }\end{array}$} \\
\hline nein & 250 & 94,0 \\
\hline ja & 16 & 6,0 \\
\hline $\begin{array}{r}\text { Niedermol. Heparin } \\
\text { prophylaktisch }\end{array}$ & & \\
\hline nein & 266 & 100,00 \\
\hline
\end{tabular}

Ein Karnofsky-Index von 60\% ist definiert als „einige Hilfestellung nötig, selbstständig in den meisten Bereichen“.

Es gab 230 Patienten (86,1\%), die einen Karnofsky-Index von 60\% oder mehr aufwiesen. Siebenunddreizig Patienten (13,9\%) hatten einen niedrigeren Karnofsky-Index. Die Verteilung stellt Tabelle 5 dar. 
Tabelle 5: Verteilung der Einteilung nach Karnofsky

\begin{tabular}{|r|r|r|}
\hline & Anzahl (n) & Anteil (\%) \\
\hline Karnofsky $>\mathbf{5 0}$ & 37 & 13,9 \\
nein & 230 & 86,1 \\
\hline
\end{tabular}

Die Ergebnisse zu dem Parameter präoperatives Trauma wird in Tabelle 6 aufgeführt. Ein Trauma lag in 151 Fällen (56,6\%) vor. Insgesamt 116 Patienten (43,4\%) konnten kein präoperatives Trauma erinnern.

Tabelle 6: Häufigkeit eines präoperativen Traumas

\begin{tabular}{|r|r|r|}
\hline & Anzahl (n) & Anteil (\%) \\
\hline Präoperatives Trauma & & \\
nein & 116 & 43,4 \\
ja & 151 & 56,6 \\
\hline
\end{tabular}

Die anamnestischen Daten zeigt Tabelle 7. Ein Hypertonus konnte bei 201 Patienten (75,3\%) festgestellt werden. Unter Diabetes mellitus litten 42 Patienten (15,7\%). Eine symptomatische Epilepsie wiesen 28 Patienten (10,5\%) auf. Eine koronare Herzkrankheit lag bei 50 Patienten (18,7\%) vor. Vierzehn Patienten (5,2\%) hatten einen Klappenersatz und 53 Patienten $(19,9 \%)$ litten unter einer Tachyarrhythmia absoluta. 
Tabelle 7: Verteilung der Vorerkrankungen

\begin{tabular}{|c|c|c|}
\hline & Anzahl (n) & Anteil (\%) \\
\hline \multicolumn{3}{|l|}{ Hypertension } \\
\hline nein & 66 & 24,7 \\
\hline ja & 201 & 75,3 \\
\hline \multicolumn{3}{|l|}{ Diabetes mellitus } \\
\hline nein & 225 & 84,3 \\
\hline ja & 42 & 15,7 \\
\hline \multicolumn{3}{|l|}{ symptomatische Epilepsie } \\
\hline nein & 239 & 89,5 \\
\hline ja & 28 & 10,5 \\
\hline \multicolumn{3}{|l|}{ koronare Herzkrankheit } \\
\hline nein & 217 & 81,3 \\
\hline ja & 50 & 18,7 \\
\hline \multicolumn{3}{|l|}{ Herzklappenersatz } \\
\hline nein & 253 & 94,8 \\
\hline ja & 14 & 5,2 \\
\hline \multicolumn{3}{|l|}{ Tachyarrhythmia absoluta } \\
\hline nein & 214 & 80,1 \\
\hline ja & 53 & 19,9 \\
\hline
\end{tabular}

\subsection{Daten zu Patienten mit Therapieerfolg}

Insgesamt 173 der Gesamtpatientenzahl (64,8\%) waren Therapieerfolge. Vierundneunzig Patienten $(35,2 \%)$ sind Therapieversager. Die Verteilung ist Tabelle $8 \mathrm{zu}$ entnehmen.

Tabelle 8: Therapieerfolg und -versagen innerhalb des Patientenkollektivs

\begin{tabular}{|r|r|r|}
\hline & Häufigkeit (n) & Anteil (\%) \\
\hline Therapieerfolg & & \\
nein & 94 & 35,2 \\
ja & 173 & 64,8 \\
\hline Total & 267 & 100,00 \\
\hline
\end{tabular}


Unter den 173 erfolgreich therapierten Patienten, konnten 106 (61,27\%) mit nur einer Minibohrlochtrepanation und 67 Patienten (38,73\%) mit bis zu drei Minibohrlochtrepanationen geheilt werden. Die Ergebnisse sind Tabelle 9 zu entnehmen.

Tabelle 9: Anzahl Minibohrlochtrepanationen unter Therapieerfolgen

\begin{tabular}{|r|r|r|}
\hline & Häufigkeit (n) & Anteil (\%) \\
\hline Therapieerfolg & & \\
mit einer Minibohrlochtrepanation & 106 & 61,27 \\
mit $\leq 3$ Minibohrlochtrepanationen & 67 & 38,73 \\
\hline Total & 173 & 100,00 \\
\hline
\end{tabular}

Insgesamt wurden 177 Patienten ausschließlich mit Minibohrlochtrepanationen behandelt. Darunter waren vier Patienten (2,26\%), die mehr als dreier Minibohrlochtrepanationen bedurften, 173 Patienten (97,74\%) wurden erfolgreich innerhalb von drei Minibohrlochtrepanationen therapiert. Dieser Verteilung stellt Tabelle 10 dar.

Tabelle 10: Therapieerfolg und -versagen unter Patienten mit alleiniger Minibohrlochtherapie

\begin{tabular}{|r|r|r|}
\hline & Häufigkeit (n) & Anteil (\%) \\
\hline Nur mit Minibohrlochtrepanation therapiert & & \\
Therapieversagen & 4 & 2,26 \\
Therapieerfolg & 173 & 97,74 \\
\hline Total & 177 & 100,00 \\
\hline
\end{tabular}

Tabelle 11 zeigt die Altersverteilung der Patienten mit Therapieerfolg und -versagen. Es wurde eine Korrelation zwischen dem Alter der Patienten und einem Therapieerfolg oder versagen untersucht. Es konnte kein statistisch signifikanter Zusammenhang ermittelt werden. 
Tabelle 11: Altersverteilung der Patienten mit Therapieerfolg und -versagen

\begin{tabular}{|c|c|c|c|c|c|}
\hline & $\begin{array}{c}\text { Therapieerfolg: } \\
\text { nein }\end{array}$ & $\begin{array}{c}\text { Anteil } \\
(\%)\end{array}$ & $\begin{array}{c}\text { Therapieerfolg: } \\
\text { ja }\end{array}$ & $\begin{array}{c}\text { Anteil } \\
(\%)\end{array}$ & p-Wert \\
\hline älter als 80 Jahre & & & & & \\
\hline nein & 64 & 68,1 & 105 & 60,7 & 0,231 \\
\hline ja & 30 & 31,9 & 68 & 39,3 & \\
\hline
\end{tabular}

Leukozyten-, Thrombozyten- und Hämoglobinwerte erwiesen sich als nicht signifikant für einen Therapieerfolg. Dabei spielt es keine Rolle, ob die Werte im oder außerhalb des Referenzbereiches liegen. Diese Ergebnisse sind in Tabelle 12 nachzuvollziehen.

Tabelle 12: Verteilung der Blutwerte unter den Patienten mit Therapieerfolg

\begin{tabular}{|c|c|c|c|c|c|}
\hline & $\begin{array}{c}\text { Therapieerfolg: } \\
\text { nein }\end{array}$ & $\begin{array}{l}\text { Anteil } \\
(\%)\end{array}$ & $\begin{array}{c}\text { Therapieerfolg: } \\
\text { ja }\end{array}$ & $\begin{array}{c}\text { Anteil } \\
(\%)\end{array}$ & p-Wert \\
\hline \multicolumn{6}{|l|}{ Leukozyten $<11\left(10^{3} / \mu\right)$} \\
\hline nein & 11 & 11,7 & 17 & 9,8 & 0,633 \\
\hline ja & 83 & 88,3 & 156 & 90,2 & \\
\hline \multicolumn{6}{|l|}{$\begin{array}{c}\text { Thrombozyten }>150 \\
\left(10^{3} / \mu\right)\end{array}$} \\
\hline nein & 7 & 7,4 & 18 & 10,4 & 0,428 \\
\hline ja & 87 & 92,6 & 155 & 89,6 & \\
\hline \multicolumn{6}{|l|}{ Hämoglobin >13,5 (g/dl) } \\
\hline nein & 54 & 57,4 & 104 & 60,1 & 0,672 \\
\hline ja & 40 & 42,6 & 69 & 39,9 & \\
\hline
\end{tabular}


Die Ergebnisse der Gerinnungswerte unter den Patienten mit Therapieerfolg und -versagen zeigt Tabelle 13. Die Tests auf signifikanten Zusammenhang zum Therapieerfolg waren ergebnislos.

Tabelle 13: Verteilung der Gerinnungswerte unter den Patienten mit Therapieerfolg

\begin{tabular}{|c|c|c|c|c|c|}
\hline & $\begin{array}{c}\text { Therapieerfolg: } \\
\text { nein }\end{array}$ & $\begin{array}{l}\text { Anteil } \\
(\%)\end{array}$ & $\begin{array}{c}\text { Therapieerfolg: } \\
\text { ja }\end{array}$ & $\begin{array}{l}\text { Anteil } \\
(\%)\end{array}$ & p-Wert \\
\hline \multicolumn{6}{|l|}{ Quick > $70(\%)$} \\
\hline nein & 20 & 21,3 & 41 & 23,7 & 0,652 \\
\hline ja & 74 & 78,7 & 132 & 76,3 & \\
\hline \multicolumn{6}{|l|}{ PTT $<37$ (sec) } \\
\hline nein & 9 & 9,6 & 17 & 9,8 & 0,947 \\
\hline ja & 85 & 90,4 & 156 & 90,2 & \\
\hline
\end{tabular}

Tabelle 14 lässt sich entnehmen, wie die Verteilung der Antikoagulanzien unter den Patienten mit Therapieerfolg ist. Keines der antikoagulativen Medikamente beeinflusst den Therapieerfolg. 
Tabelle 14: Verteilung der Antikoagulanzien unter den Patienten mit Therapieerfolg




Unter den Patienten mit Therapieerfolg gab es 97 Patienten (56,1\%) mit einem präoperativen Trauma. Bei einem p-Wert von 0,828 besteht kein statistisch signifikanter Zusammenhang (Tabelle 15).

Tabelle 15: Häufigkeit eines präoperativen Traumas unter Patienten mit Therapieerfolg

\begin{tabular}{|c|c|c|c|c|c|}
\hline & $\begin{array}{c}\text { Therapieerfolg: } \\
\text { nein }\end{array}$ & $\begin{array}{l}\text { Anteil } \\
(\%)\end{array}$ & $\begin{array}{c}\text { Therapieerfolg: } \\
\text { ja }\end{array}$ & $\begin{array}{l}\text { Anteil } \\
(\%)\end{array}$ & p-Wert \\
\hline Trauma & & & & & \\
\hline nein & 40 & 42,6 & 76 & 43,9 & 0,828 \\
\hline ja & 54 & 57,4 & 97 & 56,1 & \\
\hline
\end{tabular}

Es wurde untersucht, ob eine Korrelation zwischen einem präoperativen Karnofsky-Index über 50\% und einer erfolgreichen Therapie mittels Minibohrlochtrepanation besteht. Es konnte keine statistische Signifikanz bestätigt werden, wie Tabelle 16 zeigt.

Tabelle 16: Bewertung nach Karnofsky unter den Patienten mit Therapieerfolg

\begin{tabular}{|r|r|r|r|r|r|}
\hline & $\begin{array}{r}\text { Therapieerfolg: } \\
\text { nein }\end{array}$ & $\begin{array}{c}\text { Anteil } \\
\mathbf{( \% )}\end{array}$ & $\begin{array}{r}\text { Therapieerfolg: } \\
\mathbf{j a}\end{array}$ & $\begin{array}{c}\text { Anteil } \\
\mathbf{( \% )}\end{array}$ & p-Wert \\
\hline Karnofsky $\mathbf{5 0}$ \\
nein
\end{tabular}

Tabelle 17 zeigt den Zusammenhang zwischen Vorerkrankungen und Therapieerfolg. Keine der Vorerkrankungen korreliert mit dem Therapieerfolg. 
Tabelle 17: Häufigkeit von Vorerkrankungen unter Patienten mit Therapieerfolg

\begin{tabular}{|c|c|c|c|c|c|}
\hline & $\begin{array}{c}\text { Therapieerfolg: } \\
\text { nein }\end{array}$ & $\begin{array}{c}\text { Anteil } \\
(\%)\end{array}$ & $\begin{array}{c}\text { Therapieerfolg: } \\
\text { ja }\end{array}$ & $\begin{array}{l}\text { Anteil } \\
(\%)\end{array}$ & $\mathrm{p}$-Wert \\
\hline \multicolumn{6}{|l|}{ Hyptertension } \\
\hline nein & 23 & 24,5 & 43 & 24,9 & 0,944 \\
\hline ja & 71 & 75,5 & 130 & 75,1 & \\
\hline \multicolumn{6}{|l|}{ Diabetes mellitus } \\
\hline nein & 74 & 78,7 & 151 & 87,3 & 0,067 \\
\hline ja & 20 & 21,3 & 22 & 12,7 & \\
\hline \multicolumn{6}{|l|}{ symtomatische Epilepsie } \\
\hline nein & 80 & 85,1 & 159 & 91,9 & 0,083 \\
\hline ja & 14 & 14,9 & 14 & 8,1 & \\
\hline \multicolumn{6}{|l|}{ koronare Herzkrankheit } \\
\hline nein & 78 & 83,0 & 139 & 80,3 & 0,599 \\
\hline ja & 16 & 17,0 & 34 & 19,7 & \\
\hline \multicolumn{6}{|l|}{ Herzklappenersatz } \\
\hline nein & 87 & 92,6 & 166 & 96 & 0,234 \\
\hline ja & 7 & 7,4 & 7 & 4,0 & \\
\hline \multicolumn{6}{|l|}{ Tachyarrhythmia absoluta } \\
\hline nein & 77 & 81,9 & 137 & 79,2 & 0,594 \\
\hline ja & 17 & 18,1 & 36 & 20,8 & \\
\hline
\end{tabular}




\subsection{Auswertung der Korrelation zwischen nötigen Reoperationen und Therapieerfolg}

Bei insgesamt 161 Patienten (60,3\%) musste mindestens eine erneute Reoperation durchgeführt werden. Dies schließt sowohl erneute Minibohrloch- als auch einen Wechsel auf erweiterte Bohrlochtrepanationen ein.

Bei 106 Patienten (39,7\%) konnte bereits mit dem ersten operativen Eingriff, der Minibohrlochtrepanation, ein Therapieerfolg erreicht werden. Diese Ergebnisse sind Tabelle $18 \mathrm{zu}$ entnehmen.

Tabelle 18: Häufigkeit von Reoperationen nach initialer Minibohrlochtrepanation

\begin{tabular}{|r|r|r|}
\hline & Häufigkeit (n) & \multicolumn{2}{|c|}{ Anteil (\%) } \\
\hline Reoperation nötig & & \\
nein & 106 & 39,7 \\
$\mathrm{ja}$ & 161 & 60,3 \\
\hline Total & 267 & 100,00 \\
\hline
\end{tabular}

Die Korrelation zwischen dem Alter der Patienten und der Notwendigkeit von Reoperationen wurde untersucht. Bei einem p-Wert von 0,009 besteht ein statistisch signifikanter Zusammenhang (Tabelle 19).

Tabelle 19: Altersverteilung unter den Patienten mit Reoperation

\begin{tabular}{|c|c|c|c|c|c|}
\hline & $\begin{array}{c}\text { Nötige } \\
\text { Reoperation: } \\
\text { nein }\end{array}$ & $\begin{array}{c}\text { Anteil } \\
(\%)\end{array}$ & $\begin{array}{c}\text { Nötige } \\
\text { Reoperation: } \\
\text { ja }\end{array}$ & $\begin{array}{c}\text { Anteil } \\
(\%)\end{array}$ & p-Wert \\
\hline \multicolumn{6}{|l|}{ älter als 80 Jahre } \\
\hline nein & 57 & 53,8 & 112 & 69,9 & 0,009 \\
\hline ja & 49 & 46,2 & 49 & 30,4 & \\
\hline
\end{tabular}


Tabelle 20: Pearson-Korrelation zwischen dem Patientenalter und der Anzahl der Reoperationen

\begin{tabular}{|l|r|r|}
\hline & \multicolumn{1}{|c|}{ Alter } & \multicolumn{2}{|c|}{ Anzahl Reoperationen } \\
\hline Pearson-Korrelation & 1 & -.104 \\
\hline Sig. (2-tailed) & & .091 \\
\hline N & 267 & 267 \\
\hline
\end{tabular}

Ebenso untersucht wurde der Zusammenhang zwischen den Leukozyten-, Thrombozytenund Hämoglobinwerten und der Notwendigkeit von Reoperationen. Bei den ermittelten pWerten besteht kein statistisch signifikanter Zusammenhang (Tabelle 21).

Tabelle 21: Leukozyten-, Thrombozyten-, Hämoglobinwerte der Patienten mit Reoperation

\begin{tabular}{|c|c|c|c|c|c|}
\hline & $\begin{array}{c}\text { Nötige } \\
\text { Reoperation: } \\
\text { nein }\end{array}$ & $\begin{array}{c}\text { Anteil } \\
(\%)\end{array}$ & $\begin{array}{c}\text { Nötige } \\
\text { Reoperation: } \\
\text { ja }\end{array}$ & $\begin{array}{c}\text { Anteil } \\
(\%)\end{array}$ & p-Wert \\
\hline \multicolumn{6}{|l|}{ Leukozyten $<11\left(10^{3} / \mu\right)$} \\
\hline nein & 8 & 7,5 & 20 & 12,4 & 0,203 \\
\hline ja & 98 & 92,5 & 141 & 87,6 & \\
\hline \multicolumn{6}{|l|}{ Thrombozyten $>150\left(10^{3} / \mu\right)$} \\
\hline nein & 10 & 9,4 & 15 & 9,3 & 0,974 \\
\hline ja & 96 & 90,6 & 146 & 90,7 & \\
\hline \multicolumn{6}{|l|}{ Hämoglobin >13,5 (g/dl) } \\
\hline nein & 64 & 60,4 & 94 & 58,4 & 0,746 \\
\hline ja & 42 & 39,6 & 67 & 41,6 & \\
\hline
\end{tabular}

Tabelle 22 zeigt, dass kein statistisch signifikanter Zusammenhang zwischen den Gerinnungswerten Quick und Partielle Thromboplastinzeit und der Notwendigkeit von Reoperationen besteht. 
Tabelle 22: Gerinnungswerte der Patienten mit Reoperation

\begin{tabular}{|c|c|c|c|c|c|}
\hline & $\begin{array}{c}\text { Nötige } \\
\text { Reoperation: } \\
\text { nein }\end{array}$ & $\begin{array}{c}\text { Anteil } \\
(\%)\end{array}$ & $\begin{array}{c}\text { Nötige } \\
\text { Reoperation: } \\
\text { ja }\end{array}$ & $\begin{array}{c}\text { Anteil } \\
(\%)\end{array}$ & p-Wert \\
\hline \multicolumn{6}{|l|}{ Quick > $70(\%)$} \\
\hline nein & 22 & 20,8 & 39 & 24,2 & 0,509 \\
\hline ja & 84 & 79,2 & 122 & 75,8 & \\
\hline \multicolumn{6}{|l|}{$\begin{array}{r}\text { Partielle Thromboplastinzeit } \\
<37(\mathrm{sec})\end{array}$} \\
\hline nein & 10 & 9,4 & 16 & 9,9 & 0,892 \\
\hline ja & 96 & 90,6 & 145 & 90,1 & \\
\hline
\end{tabular}

Bei der Untersuchung der Korrelation zwischen den präoperativ eingenommenen Medikamenten zur Antikoagulation und der Notwendigkeit von Reoperationen wurde kein statistisch signifikanter Zusammenhang ermittelt, wie in Tabelle 23 ersichtlich. 
Tabelle 23: Häufigkeit von Antikoagulanzien unter Patienten mit Reoperation

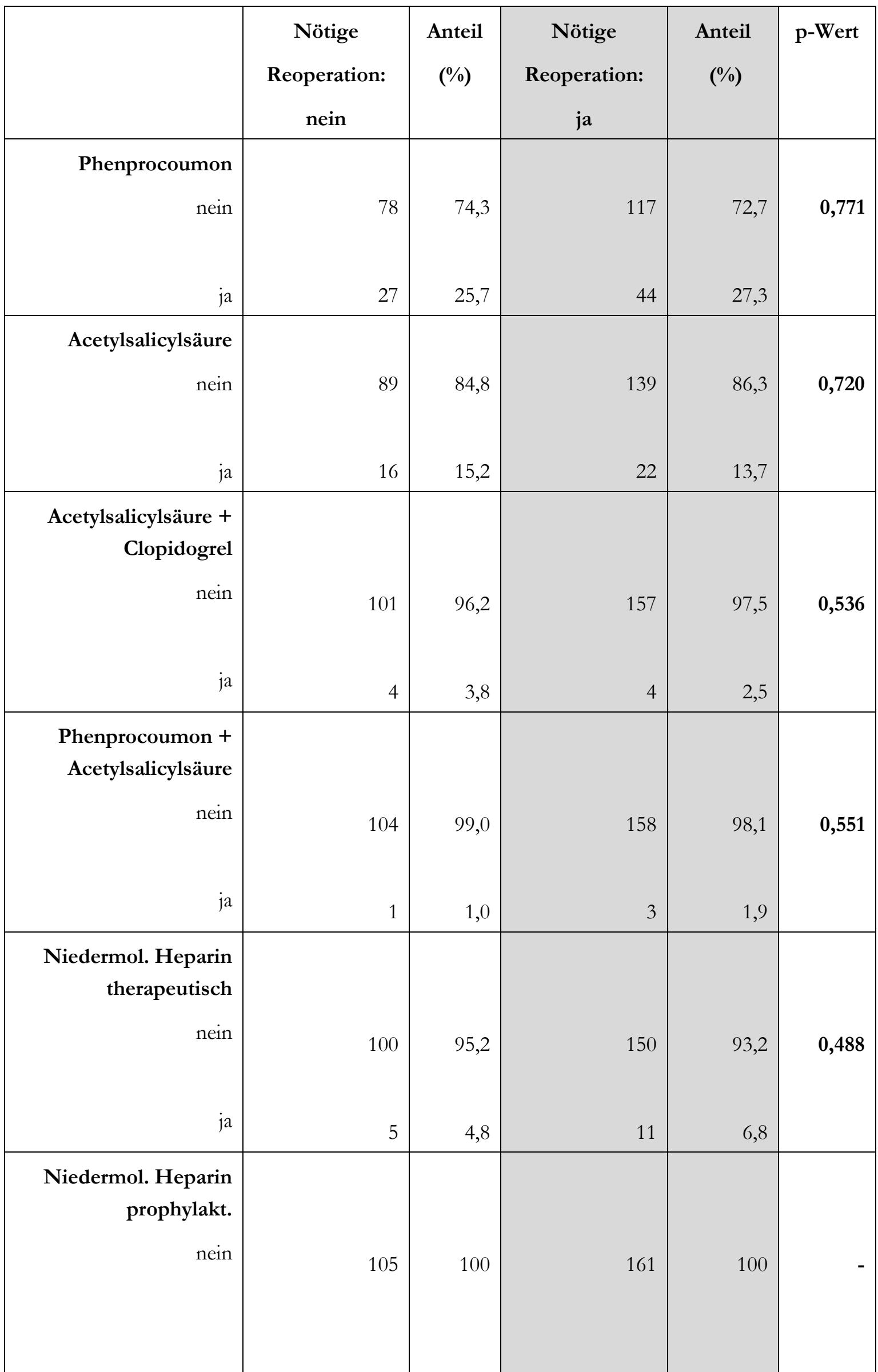


Die Untersuchung der Korrelation zwischen einem präoperativen Trauma und Reoperationen ergab einen $\mathrm{p}$-Wert von 0,075 . Es besteht kein statistisch signifikanter Zusammenhang (Tabelle 24).

Tabelle 24: Häufigkeit eines präoperativen Traumas bei Patienten mit Reoperation

\begin{tabular}{|c|c|c|c|c|c|}
\hline & $\begin{array}{c}\text { Nötige } \\
\text { Reoperation: } \\
\text { nein }\end{array}$ & $\begin{array}{c}\text { Anteil } \\
(\%)\end{array}$ & $\begin{array}{c}\text { Nötige } \\
\text { Reoperation: } \\
\text { ja }\end{array}$ & $\begin{array}{c}\text { Anteil } \\
(\%)\end{array}$ & p-Wert \\
\hline \multicolumn{6}{|l|}{ Trauma } \\
\hline nein & 39 & 36,8 & 77 & 47,8 & 0,075 \\
\hline ja & 67 & 63,2 & 84 & 52,2 & \\
\hline
\end{tabular}

Die Untersuchung des Zusammenhangs zwischen einem Karnofsky-Index über 50\% und der Notwendigkeit von Reoperationen ergab keine statistisch signifikante Korrelation. Der p-Wert liegt bei 0,910 (Tabelle 25).

Tabelle 25: Einteilung nach Karnofsky bei Patienten mit Reoperation

\begin{tabular}{|c|c|c|c|c|c|}
\hline & $\begin{array}{c}\text { Nötige } \\
\text { Reopertation: } \\
\text { nein }\end{array}$ & $\begin{array}{c}\text { Anteil } \\
(\%)\end{array}$ & $\begin{array}{c}\text { Nötige } \\
\text { Reoperation: } \\
\text { ja }\end{array}$ & $\begin{array}{c}\text { Anteil } \\
(\%)\end{array}$ & p-Wert \\
\hline \multicolumn{6}{|l|}{ Karnofsky $>50$} \\
\hline 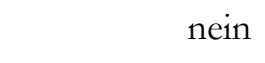 & 15 & 14,2 & 22 & 13,7 & 0,910 \\
\hline ja & 91 & 85,8 & 139 & 86,3 & \\
\hline
\end{tabular}

Hinsichtlich der Vorerkrankungen konnte jeweils keine Signifikanz zum Einsatz von Reoperationen berechnet werden. Die Daten dazu sind in Tabelle 26 aufgeführt. 
Tabelle 26: Häufigkeit von Vorerkrankungen bei Patienten mit Reoperation

\begin{tabular}{|c|c|c|c|c|c|}
\hline & $\begin{array}{c}\text { Nötige } \\
\text { Reoperation: } \\
\text { nein }\end{array}$ & $\begin{array}{c}\text { Anteil } \\
(\%)\end{array}$ & $\begin{array}{c}\text { Nötige } \\
\text { Reoperation: } \\
\text { ja }\end{array}$ & $\begin{array}{c}\text { Anteil } \\
(\%)\end{array}$ & p-Wert \\
\hline \multicolumn{6}{|l|}{ Hyptertension } \\
\hline nein & 21 & 19,8 & 45 & 28,0 & 0,131 \\
\hline ja & 85 & 80,2 & 116 & 72,0 & \\
\hline \multicolumn{6}{|l|}{ Diabetes mellitus } \\
\hline nein & 90 & 84,9 & 135 & 83,9 & 0,817 \\
\hline ja & 16 & 15,1 & 26 & 16,1 & \\
\hline \multicolumn{6}{|l|}{ symptomatische Epilepsie } \\
\hline nein & 96 & 90,6 & 143 & 88,8 & 0,649 \\
\hline ja & 10 & 9,4 & 18 & 11,2 & \\
\hline \multicolumn{6}{|l|}{ koronare Herzkrankheit } \\
\hline nein & 84 & 79,2 & 133 & 82,6 & 0,491 \\
\hline ja & 22 & 20,8 & 28 & 17,4 & \\
\hline \multicolumn{6}{|l|}{ Herzklappenersatz } \\
\hline nein & 103 & 97,2 & 150 & 93,2 & 0,151 \\
\hline ja & 3 & 2,8 & 11 & 6,8 & \\
\hline \multicolumn{6}{|l|}{ Tachyarrhythmia absoluta } \\
\hline nein & 85 & 80,2 & 129 & 80,1 & 0,990 \\
\hline ja & 21 & 19,8 & 32 & 19,9 & \\
\hline
\end{tabular}




\subsection{Daten zu Patienten mit Therapieversagen/nötigem Wechsel auf invasivere Operationsmethode}

Die Häufigkeit des Einsatzes einer invasiveren Operationsmethode, hier der erweiterten Bohrlochtrepanation und Drainageeinlage nach ein- oder mehrmaliger Minibohrlochtrepanation, zeigt Tabelle 27.

Bei 90 Patienten (33,7\%) musste nach drei Minibohrlochtrepanationen und persistierenden Symptomen eine erweiterte Bohrlochtrepanation mit Drainage durchgeführt werden.

Tabelle 27: Häufigkeit des Einsatzes invasiverer Operationsmethoden

\begin{tabular}{|r|r|r|}
\hline & Häufigkeit (n) & Anteil (\%) \\
\hline invasivere Operationsmethode nötig & & \\
nein & 177 & 66,3 \\
$\mathrm{ja}$ & 90 & 33,7 \\
\hline Total & 267 & 100,00 \\
\hline
\end{tabular}

Tabelle 28 verdeutlicht die Ursachen für Therapieversagen. Insgesamt war ein Therapieversagen bei 94 Patienten vorzufinden. Bei vier dieser Patienten (4,26\%) kam es zum Therapieversagen aufgrund der Notwendigkeit von mehr als drei Minibohrlochtrepanationen. Bei 90 Patienten (95,74\%) musste eine invasivere Operationsmethode gewählt werden.

Tabelle 28: Ursachen für Therapieversagen

\begin{tabular}{|r|r|r|}
\hline & Häufigkeit (n) & Anteil (\%) \\
\hline Therapieversagen & 4 & 4,26 \\
definiert durch $>3$ Minibohrlochtrepanationen & & \\
definiert durch invasiveres Operationsverfahren & 90 & 95,74 \\
\hline Total & 94 & 100,00 \\
\hline
\end{tabular}

Tabelle 29 verdeutlicht die Altersverteilung unter den Patienten, bei denen im Laufe der Behandlung eine invasivere OP-Methode als die Minibohrlochtrepanation angewendet wurde. Der p-Wert von 0,176 bestätigt, dass das Alter nicht korreliert mit der Notwendigkeit von invasiveren chirurgischen Eingriffen. 
Tabelle 29: Altersverteilung unter den Patienten mit invasiverer Operationsmethode

\begin{tabular}{|c|c|c|c|c|c|}
\hline & $\begin{array}{l}\text { invasivere Ope- } \\
\text { rationsmethode } \\
\text { nötig: } \\
\text { nein }\end{array}$ & $\begin{array}{c}\text { Anteil } \\
(\%)\end{array}$ & $\begin{array}{l}\text { Invasivere Ope- } \\
\text { rationsmethode } \\
\text { nötig: } \\
\text { ja }\end{array}$ & $\begin{array}{c}\text { Anteil } \\
(\%)\end{array}$ & p-Wert \\
\hline \multicolumn{6}{|l|}{ älter als 80 Jahre } \\
\hline nein & 107 & 60,5 & 62 & 68,9 & 0,176 \\
\hline ja & 70 & 39,5 & 28 & 31,1 & \\
\hline
\end{tabular}

Es wurde untersucht, ob eine Verbindung zwischen den Leukozyten-, Thrombozyten- und Hämoglobinwerten und dem Einsatz invasiverer OP-Methoden besteht.

Es hat sich gezeigt, dass dies nicht der Fall ist. Tabelle 30 sind die Details zu entnehmen.

Tabelle 30: Leukozyten-, Thrombozyten-, Hämoglobinwerte der Patienten mit invasiverer Operationsmethode

\begin{tabular}{|c|c|c|c|c|c|}
\hline & $\begin{array}{l}\text { Invasivere Ope- } \\
\text { rationsmethode } \\
\text { nötig: } \\
\text { nein }\end{array}$ & $\begin{array}{c}\text { Anteil } \\
(\%)\end{array}$ & $\begin{array}{l}\text { invasivere Ope- } \\
\text { rationsmethode } \\
\text { nötig: } \\
\text { ja }\end{array}$ & $\begin{array}{c}\text { Anteil } \\
(\%)\end{array}$ & p-Wert \\
\hline \multicolumn{6}{|l|}{ Leukozyten $<11\left(10^{3} / \mu\right)$} \\
\hline nein & 17 & 9,6 & 11 & 12,2 & 0,509 \\
\hline ja & 160 & 90,4 & 79 & 87,8 & \\
\hline \multicolumn{6}{|l|}{ Thrombozyten $>150\left(10^{3} / \mu\right)$} \\
\hline nein & 19 & 10,7 & 6 & 6,7 & 0,281 \\
\hline ja & 158 & 89,3 & 84 & 93,3 & \\
\hline \multicolumn{6}{|l|}{ Hämoglobin >13,5 (g/dl) } \\
\hline nein & 106 & 59,9 & 52 & 57,8 & 0,740 \\
\hline ja & 71 & 40,1 & 38 & 42,2 & \\
\hline
\end{tabular}


Tabelle 31 zeigt die Ergebnisse des Zusammenhanges zwischen den Gerinnungswerten und dem Einsatz invasiverer Operationsmethoden. Sowohl für den Quick-Wert als auch für die PTT konnte kein signifikanter Zusammenhang ermittelt werden.

Tabelle 31: Gerinnungswerte von Patienten mit invasiverer Operationsmethode

\begin{tabular}{|c|c|c|c|c|c|}
\hline & $\begin{array}{l}\text { invasivere Ope- } \\
\text { rationsmethode } \\
\text { nötig: } \\
\text { nein }\end{array}$ & $\begin{array}{c}\text { Anteil } \\
(\%)\end{array}$ & $\begin{array}{l}\text { invasivere Ope- } \\
\text { rationsmethode } \\
\text { nötig: } \\
\text { ja }\end{array}$ & $\begin{array}{c}\text { Anteil } \\
(\%)\end{array}$ & p-Wert \\
\hline \multicolumn{6}{|l|}{ Quick > $70(\%)$} \\
\hline nein & 41 & 23,2 & 20 & 22,22 & 0,862 \\
\hline ja & 136 & 76,8 & 70 & 77,8 & \\
\hline \multicolumn{6}{|l|}{ PTT $<37(\mathrm{sec})$} \\
\hline nein & 17 & 9,6 & 9 & 10,0 & 0,918 \\
\hline ja & 160 & 90,4 & 81 & 90,0 & \\
\hline
\end{tabular}

Die Medikamente zur Antikoagulation waren alle nicht signifikant assoziiert mit der Notwendigkeit zur Anwendung invasiverer Operationsmethoden. Diese Zusammenhänge sind in Tabelle 32 zusammengefasst. 
Tabelle 32: Häufigkeit von Antikoagulanzien unter den Patienten mit invasiverer Operationsmethode

\begin{tabular}{|c|c|c|c|c|c|}
\hline & $\begin{array}{l}\text { invasivere Ope- } \\
\text { rationsmethode } \\
\text { nötig: } \\
\text { nein }\end{array}$ & $\begin{array}{c}\text { Anteil } \\
(\%)\end{array}$ & $\begin{array}{l}\text { Invasivere Ope- } \\
\text { rationsmethode } \\
\text { nötig: } \\
\text { ja }\end{array}$ & $\begin{array}{l}\text { Anteil } \\
(\%)\end{array}$ & p-Wert \\
\hline \multicolumn{6}{|l|}{ Phenprocoumon } \\
\hline nein & 130 & 73,9 & 65 & 72,2 & 0,775 \\
\hline ja & 46 & 26,1 & 25 & 27,8 & \\
\hline \multicolumn{6}{|l|}{ Acetylsalicylsäure } \\
\hline nein & 152 & 86,4 & 76 & 84,4 & 0,672 \\
\hline ja & 24 & 13,6 & 14 & 15,6 & \\
\hline \multicolumn{6}{|l|}{$\begin{array}{r}\text { Acetylsalicylsäure }+ \\
\text { Clopidogrel }\end{array}$} \\
\hline nein & 170 & 96,6 & 88 & 97,8 & 0,592 \\
\hline ja & 6 & 3,4 & 2 & 2,2 & \\
\hline \multicolumn{6}{|l|}{$\begin{array}{l}\text { Phenprocoumon + } \\
\text { Acetylsalicylsäure }\end{array}$} \\
\hline nein & 173 & 98,3 & 89 & 98,9 & 0,707 \\
\hline ja & 3 & 1,7 & 1 & 1,1 & \\
\hline $\begin{array}{r}\text { Niedermol. Heprain } \\
\text { therapeut. } \\
\text { nein }\end{array}$ & 166 & 94,3 & 84 & 93,3 & 0,749 \\
\hline ja & 10 & 5,7 & 6 & 6,7 & \\
\hline $\begin{array}{r}\text { Niedermol. Heparin } \\
\text { prophylakt. } \\
\text { nein }\end{array}$ & 176 & 100,00 & 90 & 100,00 & - \\
\hline
\end{tabular}


Der Zusammenhang zwischen einem präoperativen Trauma und der Häufigkeit von invasiveren chirurgischen Eingriffen wurde untersucht. Bei einem p-Wert von 0,979 besteht keine statistisch signifikante Korrelation (Tabelle 33).

Tabelle 33: Häufigkeit von präoperativem Trauma bei Patienten mit invasiverer Operationsmethode

\begin{tabular}{|r|r|r|r|r|r|}
\hline & $\begin{array}{c}\text { Invasivere Ope- } \\
\text { rationsmethode } \\
\text { nötig: } \\
\text { nein }\end{array}$ & $\begin{array}{c}\text { Anteil } \\
\mathbf{( \% )}\end{array}$ & $\begin{array}{c}\text { invasivere Ope- } \\
\text { rationsmethode } \\
\text { nötig: } \\
\text { Trauma }\end{array}$ & $\begin{array}{c}\text { Anteil } \\
\mathbf{( \% )}\end{array}$ & p-Wert \\
\hline nein & 77 & 43,5 & 39 & 43,3 & $\mathbf{0 , 9 7 9}$ \\
ja & 100 & 56,5 & 51 & 56,7 & \\
\hline
\end{tabular}

Für den Zusammenhang zwischen einem Karnofsky-Index über 50\% und der Notwendigkeit invasiverer Operationsmethoden wurde ein p-Wert von 0,354 ermittelt. Es besteht keine statistisch signifikante Korrelation (Tabelle 34).

Tabelle 34: Einteilung nach Karnofsky-Index unter Patienten mit invasiverer Operationsmethode

\begin{tabular}{|r|r|r|r|r|r|}
\hline & $\begin{array}{c}\text { Invasivere Ope- } \\
\text { rationsmethode } \\
\text { nötig: } \\
\text { nein }\end{array}$ & $\begin{array}{c}\text { Anteil } \\
\mathbf{( \% )}\end{array}$ & $\begin{array}{c}\text { Invasivere Ope- } \\
\text { rationsmethode } \\
\text { nötig: } \\
\text { ja }\end{array}$ & $\begin{array}{c}\text { Anteil } \\
\mathbf{( \% )}\end{array}$ & p-Wert \\
\hline Karnofsky > 50 & 27 & 15,3 & 10 & 11,1 & $\mathbf{0 , 3 5 4}$ \\
nein & 150 & 84,7 & 80 & 88,9 & \\
\hline
\end{tabular}

Tabelle 35 erfasst die untersuchten Vorerkrankungen der Patienten mit invasiveren Operationsmethoden. Einzig für den Parameter Diabetes mellitus konnte ein signifikanter Zusammenhang zur Notwendigkeit von invasiveren Operationsmethoden identifiziert werden. 
Tabelle 35: Häufigkeit der Vorerkrankungen unter den Patienten mit invasiverer Operationsmethode






\subsection{Daten zu Komplikationen}

Als Komplikation hat sich bei acht Patienten (3\%) ein postoperatives Empyem ergeben. In der statistischen Auswertung ergab sich keine signifikante Korrelation zwischen dem Alter der Patienten, den Blut- oder Gerinnungswerten, dem Vorliegen eines Traumas, dem Karnofsky-Index oder den Vorerkrankungen. Aufgrund der vorliegenden Daten muss jedoch von einem signifikanten Zusammenhang zwischen der therapeutischen Einnahme von niedermolekularem Heparin und dem Auftreten eines postoperativen Empyems ausgegangen werden. Es wurde ein p-Wert von 0,022 berechnet. 


\section{Diskussion}

Das Krankheitsbild des cSDH ist seit vielen Jahrhunderten bekannt und tritt vor allem im höheren Alter auf. Es stellt die häufigste intrakranielle Blutung dar.

Ein Einriss der Brückenvenen wurde als Ursache eindeutig definiert (Chen und Levy 2000). Weitere Faktoren, wie zum Beispiel ein erhöhter osmotischer Druck, führen zu einer Volumenzunahme des initial oft sehr schmalen Hämatoms. Trotz der Häufigkeit herrscht immer noch Uneinigkeit, mit welchem operativen Verfahren das $\mathrm{cSDH}$ am besten zu therapieren ist (Rohde et al. 2002).

Aktuelle Therapieverfahren sind die Minibohrlochtrepanation, die erweiterte Bohrlochtrepanation mit oder ohne Drainageeinlage sowie die Kraniotomie, wobei zunehmendes Interesse für das minimal-invasive Verfahren der Minibohrlochtrepanation aufkommt und sich bei der Kraniotomie zunehmend die Frage nach einer Übertherapie stellt. Aktuell kommt hauptsächlich die erweiterte Bohrlochtrepanation mit oder ohne Drainageeinlage zur Anwendung (Ducruet et al. 2012). In der Suche nach dem besten Therapieverfahren spielen die Effektivität, die operationsassoziierte Morbidität und Mortalität sowie die Rezidivquote die entscheidende Rolle.

Es wurden 267 Patienten mit cSDH eingeschlossen, die im Zeitraum von 2007-2011 in der Klinik für Neurochirurgie der Georg-August-Universität Göttingen behandelt wurden. Die Operationsdokumentation ist Grundlage für die Datenerhebung, zusammen mit den Arztbriefen und anamnestischen Daten der Patienten. Patienten mit gleichzeitig vorliegendem rechts- und linksseitigen cSDH wurden als zwei separate Fälle angesehen. Es erfolgte die Dokumentation von Alter, Geschlecht, Anzahl und Verfahren aller erfolgten Operationen, anamnestischen Angaben zu internistischen Vorerkrankungen und Traumata, präoperativen Laborparametern aus Blutbild und Gerinnung und präoperativer Einnahme antikoagulativer Medikamente. Alle Patienten wurden initial mit einer Minibohrlochtrepanation therapiert. Die Operation orientiert sich an dem Verfahren von Reinges et al. (2000) mit einem Bohrloch von 3-4 mm ohne Anlage einer Drainage. Das Drainieren erfolgte über eine Venenverweilkanüle. Als Therapieerfolg wurden die Fälle angesehen, die mit maximal drei Minibohrlochtrepanationen geheilt werden konnten. Die Patienten, die mehr als drei Minibohrlochtrepanationen erhielten oder bei denen ein Wechsel auf eine erweiterte Bohrlochtrepanation mit oder ohne Drainageeinlage erfolgen musste, werden in die Gruppe „Therapieversagen“ eingeordnet.

Es können folgende Ergebnisse zusammengefasst werden: 177 Patienten wurden ausschließlich mit Minibohrlochtrepanationen therapiert. Darunter konnten 173 Patienten erfolgreich mit maximal drei Minibohrlochtrepanationen therapiert werden. Daraus ergibt sich eine Erfolgsquote von 97,74\% für die Patienten, die für die ausschließliche Therapie mit Minibohrlochtrepanationen geeignet waren. 
Keiner der untersuchten präoperativen Parameter korrelierte mit einem besseren oder schlechteren Therapieerfolg.

Unter den 173 erfolgreich therapierten Patienten war bei 106 Patienten $(61,27 \%)$ nur eine Operation nötig, bei 67 Patienten (38,73\%) erfolgten zwei oder drei Minibohrlochtrepanationen.

Innerhalb der minibohrlochtherapierten Patienten ergibt sich daraus eine Rezidivquote von $37,9 \%$ (67 von 177 Patienten).

Unter den 94 Therapieversagern befinden sich vier Patienten, bei denen mehr als drei Minibohrlochtrepanationen nötig waren, bei 90 Patienten war ein Wechsel auf ein invasiveres Operationsverfahren notwendig.

Es konnte herausgearbeitet werden, dass das Alter der Patienten die Notwendigkeit von Reoperationen beeinflusst. Patienten über 80 Jahre benötigten signifikant häufiger Reoperationen als Patienten unter 80 Jahre. Alle anderen Parameter nahmen auf die Anzahl der Reoperationen keinen Einfluss.

Die Notwendigkeit invasiverer Operationsverfahren, hier ausschließlich der erweiterten Bohrlochtrepanation mit Drainageeinlage, wird nur durch den Faktor Diabetes mellitus beeinflusst. Es zeigte sich, dass Patienten mit Diabetes mellitus häufiger eine invasivere Operationsmethode brauchten als Patienten ohne diese Grunderkrankung.

Die Fragestellung der Untersuchung beleuchtet Parameter für ein optimales Therapieansprechen einer Minibohrlochtrepanation. Die Effektivität von Minibohrlochtrepanationen wird in der Literatur noch kritisch gesehen. Gerade in Hinblick auf die Therapie von Hochrisikopatienten sollen Übertherapien vermieden werden.

Die Analyse unserer Daten erlaubt keine vollständige Identifikation der prädisponierenden Faktoren für einen Therapieerfolg mittels Minibohrlochtrepanation. Keiner der Parameter zeigte eine Korrelation zum Therapieerfolg. Es können lediglich Teilbereiche der Fragestellung Beantwortung finden. So kann für die Notwendigkeit von Reoperationen die Patientengruppe der über 80-Jährigen als prädisponiert angegeben werden.

Ebenso fanden invasivere Operationsmethoden vermehrten Einsatz unter an Diabetes mellitus erkrankten Patienten.

In vorangegangenen Studien lag das Augenmerk vornehmlich auf der reinen Auswertung von Erfolgs- und Rezidivraten der einzelnen Operationsverfahren. Die vorliegende Untersuchung beleuchtet zusätzlich mehrere präoperative Parameter und ihren Einfluss auf den Therapieerfolg von Minibohrlochtrepanationen. Sie zeichnet sich durch eine erhöhte Anzahl von untersuchten Faktoren im Vergleich zu vorhandenen Studien aus. Zu anamnestischen Daten und Vorerkrankungen in Bezug auf das Outcome nach chirurgischer Therapie des cSDH gibt es bisher nur geringes Datenmaterial. Die vorliegende Arbeit schließt eine Vielzahl präoperativer Parameter ein, wodurch versucht wurde, eine erste Antwort sowohl zu 
Laborparametern und Gerinnungswerten als auch zu internistischen Vorerkrankungen in Bezug auf das Outcome nach Minibohrlochtrepanation zu geben. Die Bestimmung verschiedener Laborparameter wird im Rahmen der präoperativen Routineuntersuchung vorgenommen. Es ist daher naheliegend, Zusammenhänge zwischen ihnen und dem Gesundheitszustand der Patienten zu beobachten und zu interpretieren. Hier ist eine Überlegenheit in Bezug zu vorhandenen Untersuchungen zu sehen, die diese Daten nicht in die Auswertung eingeschlossen haben. Darüber hinaus schließt unsere Studie bereits mehrfach untersuchte Parameter wie das präoperative Trauma mit ein und kann so die Ergebnisse vorhandener Studien untermauern. Trotz der erhöhten Zahl an integrierten Parametern, sind Vergleiche zum Aufbau und Design anderer Untersuchungen möglich, wie der von Reinges et al. (2000). Der Zeitraum der Datenerhebung zwischen 2007 und 2011 kann als angemessen eingeschätzt werden.

Insgesamt wurde mit 267 Patienten ein größeres Kollektiv untersucht, als in vorherigen Studien. Reinges et al. (2000) untersuchten 118 Patienten, Horn et al. (2006) 79 Patienten, Yadav et al. (2013) schlossen 50 Patienten in ihre Untersuchung ein und Stanisic et al. (2005) 121 Patienten. Die Aussagekraft kann durch die höhere Fallzahl gesteigert werden.

Die aktuelle Studienlage zeigt unterschiedliche Ansätze, um Prädiktoren und Risikofaktoren der verschiedenen Operationsverfahren zu analysieren. Die Einflussfaktoren auf den Erfolg von Minibohrlochtrepanationen stehen in der bisherigen Literatur noch wenig im Fokus der Untersuchungen. Unsere Daten haben keinen Prädiktor für einen Therapieerfolg mittels Minibohrlochtrepanation ergeben. Es liegen Studien vor, die ebenfalls keinen Zusammenhang einzelner Parameter zum Therapieerfolg bestätigen. So konnten die Autoren Mori und Maeda (2001) keinen Zusammenhang zwischen präoperativen Medikamenten, vor allem Vitamin-K-Antagonisten, und dem Outcome nachweisen. Sie therapierten 500 Patienten mit erweiterter Bohrlochtrepanation und Drainageeinlage. Ebenso fanden Franke et al. (1990) und Mattle et al. (1989) keine Korrelation zwischen dem Ausmaß an subduraler Blutung und der INR.

Reinges et al. (2000) konnten in ihrem Kollektiv von 118 Patienten mit bis zu 5 Minibohrlochtrepanationen 92\% der Patienten mit unilateralem und mit bis zu 10 Minibohrlochtrepanationen 95\% der Patienten mit bilateralem cSDH erfolgreich therapieren. In unserem Kollektiv konnte durch Minibohrlochtrepanationen mit maximal drei Eingriffen zu 97,74\% eine erfolgreiche Therapie erreicht werden. Im Vergleich zur Untersuchung von Reinges et al. (2000) ist das eine deutliche Reduktion der Anzahl an Operationen bei vergleichbaren Erfolgsraten. Horn et al. (2006) zogen einen Vergleich der Effektivität von Minibohrlochund erweiterten Bohrlochtrepanationen. Bei 55 Patienten kam eine Minibohrlochtrepanation zum Einsatz, bei 24 Patienten eine erweiterte Bohrlochtrepanation. Die Ergebnisse waren vergleichbar. Stanisic et al. (2005) therapierten 99 Patienten und $121 \mathrm{cSDH}$ mit erweiterten Bohrlochtrepanationen. Eine Drainage wurde in 67,8\% angelegt. In 82,6\% der Fälle reichte 
der Initialeingriff zur Heilung aus, 95,9\% der Patienten waren innerhalb von 2 Eingriffen geheilt und in 1,6\% der Fälle wurde ein dritter Eingriff nötig.

Rezidive sind bei allen Operationsverfahren nicht selten. In der vorliegenden Studie wurde für die Patienten, die ausschließlich mit Minibohrlochtrepanation therapiert wurden, eine Rezidivrate von 37,9\% ermittelt. Mehrere Untersuchungen haben sich ebenfalls mit der Problematik der Rezidive und rezidivbegünstigender Faktoren auseinandergesetzt. Sie beschreiben vergleichsweise geringere Rezidivraten. Escosa Baé et al. (2011) konnten eine Rezidivrate von 12\% für die Minibohrlochtrepanation ermitteln. Diese Rate war jedoch vergleichbar mit ihren Ergebnissen zur Rezidivrate von erweiterten Bohrlochtrepanationen. Sie geben die durchschnittliche Rezidivrate nach Minibohrlochtrepanationen mit 9,8-33\% an. Die Rezidivrate für die erweiterte Bohrlochtrepanation liegt nach Santarius et al. (2009) mit Drainage bei $9,3 \%$ und ohne Drainage bei $24 \%$.

Ebenso von Interesse ist die Mortalitätsrate nach Minibohrlochtrepanation, die nach Rohde et al. (2002) 13,3\%, nach Horn et al. (2006) 7\% und nach Reinges et al. (2000) 1\% beträgt. In der vorliegenden Untersuchung fand sich keine Mortalität.

Unsere Daten konnten den Parameter Diabetes mellitus als prädisponierend für den Einsatz invasiverer Operationsmethoden eruieren. Matsumoto et al. (1999) beschäftigten sich ebenfalls mit dem Einfluss des Diabetes mellitus und geben wiederum eine höhere Reoperationsrate nach erweiterter Bohrlochtrepanation mit Drainageeinlage an. Zumkeller et al. (1997) sprechen von einer erhöhten Letalität und einem schlechteren Outcome bei Diabetikern nach erweiterter Bohrlochtrepanation mit Drainageeinlage.

Es konnte herausgearbeitet werden, dass Patienten über 80 Jahre eine erhöhte Tendenz aufweisen, mehr als eine Operation zu benötigen. Es ist bisher nur ein Zusammenhang zwischen dem Alter der Patienten und einer zunehmenden Morbidität und Mortalität bei cSDH wissenschaftlich bestätigt (Gelabert-Gonzalez et al. 2005, van Havenbergh et al. 1996). In Bezug auf die Inzidenz sehen Mori und Maeda (2001) den Gipfel im achten Lebensjahrzehnt. Reinges et al. (2000) empfehlen Minibohrlochtrepanationen als Erstmaßnahme, vor allem bei Patienten mit reduziertem Allgemeinzustand.

Die Ergebnisse zeigen auf, dass die Minibohrlochtrepanation als minimal-invasives Therapieverfahren für cSDH ihre Berechtigung hat und lässt eine Empfehlung zum vermehrten Einsatz als Methode der ersten Wahl ableiten.

Es ist anhand der Ergebnisse davon auszugehen, dass auch ältere Patienten, die oftmals mehrere Vorerkrankungen aufweisen, von der gewählten Methode profitieren. Es kann also auch für diese Patienten eine klare Empfehlung zur Minibohrlochtrepanation ausgesprochen werden, es ist jedoch darauf hinzuweisen, dass diese Patienten ein erhöhtes Risiko für Reoperationen aufweisen. Die Patientengruppe der Diabetiker sollte wissenschaftlich weiter beleuchtet werden, um den Einsatz der Minibohrlochtrepanationen noch gezielter zu gestalten. Hier besteht die Tendenz, invasivere Operationsmethoden anwenden zu müssen. Ducruet et al. 
(2012) zeigten auf, dass es noch beträchtliche Unstimmigkeiten über die beste Therapie des cSDH gibt. Die vorliegenden Ergebnisse können die Entscheidung zur Minibohrlochtrepanation bei Vorliegen eines cSDH erleichtern. Die Aussage von Zhang et al. (2012), Minibohrlochtrepanation als sicher und effektiv einzuschätzen wird unterstützt, auch in Hinblick auf ältere und vorerkrankte Patienten.

Es liegen wiederum auch einige Limitationen der vorgelegten Studie vor. Die Ergebnisse beziehen sich auf die Gesamtpatientenzahl von 267. Anhand der Fragestellung und Definition bezieht sich der Therapieerfolg jedoch nur auf die 177 Patienten, die mit Minibohrlochtrepanation behandelt wurden. Aufgrund dessen muss die Bewertung der Ergebnisse immer dem entsprechenden Teilkollektiv zugeordnet werden. Das macht eine Vergleichbarkeit der Gesamtergebnisse zu bestehenden Untersuchungen unübersichtlicher. Nicht zu allen Patienten war die Anamnese gleichermaßen ausführlich erfassbar. Die Dokumentation vorangegangener Traumata ist abhängig vom Erinnerungsvermögen der häufig hochbetagten Patienten. Ebenso hängt die Datenlage einer retrospektiven Analyse von der Güte der Daten in den vorliegenden Krankenblättern und Operationsdokumentationen ab. Es erfolgte auch keine Erfassung der behandelnden Chirurgen, denen eine gewisse Beeinflussung der Ergebnisse hätte zugeschrieben werden können.

In der statistischen Auswertung kam die multivariate Analyse nicht zum Einsatz. Da sich nicht mehrere der Parameter als signifikant herausgestellt haben, war keine Berechnung der Korrelation untereinander sinnvoll.

Die Tatsache, dass viele präoperative Parameter eingeschlossen wurden, führt dazu, dass ein Vergleich zu vorhandenen Untersuchungen schwerer möglich ist. Ebenso erfolgte keine Unterscheidung von uni- und bilateralen Hämatomen wie bei Reinges et al. (2000). Patienten, die zum gleichen Zeitpunkt beidseitige cSDH aufwiesen, wurden als zwei getrennte Patientenfälle gewertet. Es liegt die Vermutung nahe, dass bilaterale cSDH ein schlechteres Outcome haben, weil sie ein größeres Areal betreffen als einseitige. Allerdings konnte das in der Literatur bisher nicht bestätigt werden (Penchet et al. 1998, Tsutsumi et al. 1997).

Ergänzend zu den erzielten Ergebnissen wäre von großem Interesse gewesen, die neuroradiologische Auswertung der cSDH einzuschließen. Leider lag kein ausreichendes Datenmaterial zu den Patienten vor, die erfolgreich mit Minibohrlochtrepanationen therapiert werden konnten.

Es erfolgte keine Unterscheidung zwischen Typ-1- und Typ-2-Diabetikern und keine Dokumentation der HbA1c-Werte.

Weiterführende Untersuchungen zu prädisponierenden Faktoren, die es ermöglichen, vor der Primäroperation eine Prognose für das Outcome des Patienten nach Minibohrlochtrepanation auszusprechen, sind wünschenswert. Gegebenenfalls bestehen Kausalzusammenhänge zwischen einzelnen Parametern und dem Einfluss auf ein optimales Therapieansprechen. 
Da keiner der untersuchten Parameter signifikant mit dem Therapieerfolg korrelierte, ist die Aussagekraft der Ergebnisse auf weitere Forschung angewiesen, bis eine uneingeschränkte Empfehlung zur Minibohrlochtrepanation als Methode der ersten Wahl gegeben werden kann.

Fasst man zusammen, so erwies sich die Minibohrlochtrepanation in unserer Untersuchung als gute Behandlungsmethode, gerade in Hinblick auf Hochrisikopatienten. Weiterer Untersuchungs- und Forschungsbedarf besteht in der Analyse des Zusammenhangs zwischen anamnestischen und laborchemischen Paramatern und dem präoperativen Erscheinungsbild der cSDH im cCT. Es wäre denkbar, einen Zusammenhang zu Therapieerfolgen zu hinterfragen.

Ebenso von Interesse wäre eine Analyse von Hämatomflüssigkeit, um beide vorliegenden Untersuchungen von Stanisic et al. (2012a/ 2012b) zu ergänzen. Gegebenenfalls lassen sich Inhaltsstoffe eruieren, die ein Therapieansprechen oder -versagen vorhersagen könnten. 


\section{$6 \quad$ Zusammenfassung}

Das nötige Ausmaß der chirurgischen Therapie der chronischen Subduralhämatome wird in der Literatur nach wie vor kontrovers diskutiert. Es gibt eine Vielzahl von Untersuchungen zu möglichen Operationsverfahren. Die Erkenntnisse zur Minibohrlochtrepanation sollen durch die vorgelegte Studie erweitert werden.

Dazu werden präoperative anamnestische Faktoren beleuchtet, die mögliche Prädiktoren für ein Therapieansprechen oder -versagen nach Minibohrlochtrepanation darstellen könnten. Ziel ist, präoperativ die Patienten zu identifizieren, die von der genannten Operationsmethode profitieren werden.

Einbezogen wurden insgesamt 267 Patienten, die im Zeitraum von Anfang 2007 bis Ende 2011 in der Universitätsklinik Göttingen operiert wurden. Darunter befanden sich 177 Patienten, die keine andere Operationsmethode als Minibohrlochtrepanationen erhielten. Alle anamnestischen Daten zu Geschlecht, Alter, Anzahl der erfolgten operativen Eingriffe, präoperativer Medikation, Vorerkrankungen und Blutwerten wurden den Arzt- und Operationsberichten entnommen. Sie wurden in einer Tabelle dokumentiert.

Berechnet wurde jeweils der Einfluss eines jeden Parameters auf die Rezidivrate.

Als Therapieerfolg wurde eine maximale Anzahl von drei Minibohrlochtrepanationen zur Erzielung einer klinischen Heilung definiert.

Die Erfolgsquote für alleinig mit Minibohrloch therapierte Patienten lag bei 97,74\%.

Die Methode der Minibohrlochtrepanation wird als minimal-invasive Erstmaßnahme empfohlen. Auch ältere und vorerkrankte Patienten profitieren von einer Operation mittels Minibohrloch. Das Verfahren ist unseres Erachtens weiterhin Methode der ersten Wahl, die keiner Patientengruppe vorenthalten werden soll. Bei Patienten mit Diabetes mellitus hat sich eine erhöhte Prädisposition für ein Rezidiv gezeigt. 


\section{$7 \quad$ Literaturverzeichnis}

Ansell J, Hirsh J, Poller L, Bussey H, Jacobson A, Hylek E (2004): The pharmacology and management of vitamin $\mathrm{k}$ antogonists. Chest $126,204 \mathrm{~S}-233 \mathrm{~S}$

Becker T, Dauch W, Huffmann G (1988): Das chronische subdurale Hämatom. Dtsch Med Wochenschr 113 , 439-442

Camel M, Grubb RL Jr (1986): Treatment of chronic subdural hematoma by twist-drill craniotomy with continuous catheter drainage. J Neurosurg $\underline{65}, 183-187$

Cenic A, Bhandari M, Reddy K (2005): Management of chronic subdural hematoma: a national survey and literature review. Can J Neurol Sci $\underline{32}$, 501-506

Chen JC, Levy ML (2000): Causes, epidemiology, and risk factors of chronic subdural hematoma. Neurosurg Clin N Am 11, 399-406

Drake ME Jr. (1993): Mozart`s chronic subdural hematoma. Neurology 43, 2400-2403

Drapkin AJ (1991): Chronic subdural hematoma: pathophysiological basis for treatment. Br J Neurosurg $\underline{5}, 467-473$

Ducruet AF, Grobelny BT, Zacharia BE, Hickman ZL, DeRosa PL, Anderson K, Sussman E, Carpenter A, Connolly Jr. ES (2012): The surgical management of chronic subdural hematoma. Neurosurg Rev $\underline{35}, 155-169$

El Kadi, H, Miele VJ, Kaufmann HH (2000): Prognosis of chronic subdural hematomas. Neurosurg Clin N Am 11, 553-567

Escosa Baé M, Wessling H, Salca HC, de las Heras Echeverriá P (2011): Use of twist-drill craniostomy with drain in evacuation of chronic subdural hematomas: independent predictors of recurrence. Acta Neurochir 153, 1097-1103 
Franke CL, de Jonge J, van Swieten JC, Op de Coul AA, van Gijn J (1990): Intracerebral hematomas during anticoagulant treatment. Stroke 21, 726-730

Frati A, Salvati M, Mainiero F, Ippoliti F, Rocchi G, Raco A., Caroli E, Cantore G, Delfini $\mathrm{R}$ (2004): Inflammation markers and risk factors for recurrence in 35 patients with a posttraumatic chronic subdural hematoma: a prospective study. J Neurosurg $\underline{100}$, 24-32

Gelabert-González M, Iglesias-Pais M, García-Allut A, Martínez-Rumbo R (2005): Chronic subdural haematoma: surgical treatment and outcome in 1000 cases. Clin Neurol Neurosurg 107, 223-229

Hamilton MG, Frizzell JB, Tranmer BI (1993): Chronic subdural haematoma: the role of craniotomy reevaluated. Neurosurgery $\underline{33}, 67-72$

Hanley JP (2004): Warfarin reversal. J Clin Pathol 푸, 1132-1139

Harders A, Weigel K, Gilsbach J, Eggert HR: Follow-up and results of external drainage therapy of chronic subdural hematomas. In: Schiefer W, Klinger M, Brock M (Hrsg.) Brain abscess and meningitis. Subarachnoid hemorrhage: timing problems. Advances in Neurosurgery, Bd. 9. Springer-Verlag, Berlin Heidelberg 1981, 388-390

Horn EM, Feiz-Erfan I, Bristol RE, Spetzler RF, Harringtion TR (2006): Bedside twist-drill craniostomy for chronic subdural hematoma: a comparative study. Surg Neurol $\underline{65}, 150-153$

Ito H, Yamamoto S, Komai T, Mizukoshi H (1976): Role of local hyperfibrinolysis in the etiology of chronic subdural hematoma. J Neurosurg $\underline{45}, 26-31$

Karnofsky DA, Burchenal JH: The clinical evaluation of chemotherapeutic agents in cancer. Evaluation in chemotherapeutic agents. In: MacLeod CM (Hrsg.): Evalutation of Chemotherapeutic Agents. Columbia University Press, New York 1949, 191-205

König SA, Schick U, Döhnert J, Goldammer A, Vitzthum HE (2003): Coagulopathy and outcome in patients with chronic subdural haematoma. Acta Neurol 107, 110-116 
Konstanian V, Choi JC, Liker MA, Go JL, Zee CS (2000): Computer tomographic characteristics of chronic subdural hematomas. Neurosurg Clin N Am 11, 479-489

Kotwica Z, Brzezinski J (1991): Chronic subdural hematoma treated by burr holes and closed system drainage: personal experience in 131 patients. Br J Neurosurg $\underline{5}$, 461-465

Kuroki T, Katsume M, Harada N, Yamazaki T, Aoki K, Takasu N (2001): Strict closedsystem drainage for treating chronic subdural hematoma. Acta Neurochir (Wien) $\underline{143}, 1041$ 1044

Markwalder TM, Steinsiepe KF, Rohner M, Reichenbach W, Markwalder H (1981): The course of chronic subdural hematomas after burr-hole craniostomy and closed-system-drainage. J Neurosurg $\underline{5}, 390-396$

Matsumoto K, Akagi K, Abekura M, Ryujin H, Ohkawa M, Iwasa N, Akiyama C (1999): Recurrence factors of chronic subdural hematomas after burr-hole craniostomy and closedsystem drainage. Neurol Res $\underline{21}, 277-280$

Mattle H, Kohler S, Huber P, Rohner M, Steinsiepe KF (1989): Anticoagulation-related intracranial extracerebral haemorrhage. J Neurol Neurosurg Psychiatry $\underline{52}$, 829-837

Meese W, Kluge W, Grumme T, Hopfenmüller W (1980): CT Evacuation of the CSF Space of healthy persons. Neuroradiology $\underline{19}, 131-136$

Mori K, Maeda M (2001): Surgical treatment of chronic subdural hematoma in 500 consecutive cases: clinical characteristics, surgical outcome, complications, and recurrence rate. Neurol Med Chir (Tokyo) 41, 371-381

Murakami H, Hirose Y, Sagoh M, Shimizu K, Kojima M, Gotoh K, Mine Y, Hayashi T, Kawase T (2002): Why do chronic subdural hematomas continue to grow slowly and not coagulate? Role of thrombomodulin in the mechanism. J Neurosurg $\underline{96}, 877-884$ 
Nagata K, Asano T, Basugi N, Tango T, Takakura K (1989): Studies on the operative factors affecting the reduction of chronic subdural hematoma, with special reference to the residual air in the hematoma cavity. No Shinkei Geka 17, 15-20 (Artikel auf Japanisch)

Nakaguchi H, Tanishima T, Yoshimasu N (2000): Relationship between drainage catheter location and postoperative recurrence of chronic subdural hematoma after burr-hole irrigation and closed-system drainage. J Neurosurg $\underline{93}$, 791-795

Nakaguchi H, Tanishima T, Yoshimasu N (2001): Factors in the natural history of chronic subdural hematomas that influence their postoperative recurrence. J Neurosurg $\underline{95}$, 256-262

Nomura S, Kashiwagi S, Fujisawa H, Ito H, Nakamura K (1994): Characterization of hyperfibrinolysis in chronic subdural hematomas by SDS-PAGE and immonublot. J Neurosurg 81, 910-913

Oishi M, Makoto T, Tamatani S, Kitazawa T, Saito M (2001): Clinical factors of recurrent chronic subdural hematoma. Neurol Med Chir (Tokyo) 41, 382-386

Okada Y, Akai T, Okamoto K, Iida T, Takata H, Iizuka H (2002): A comparative study of the treatment of chronic subdural hematoma- burr hole drainage versus burr-hole irrigation. Surg Neurol 57, 405-409

Penchet G, Loiseau H, Castel JP (1998): Chronic bilateral subdural hematomas. Neurochirurgie 44, 247-252 (Artikel auf Französisch)

Piotrowski WP, Krombholz-Reindl MA (1996): Operationsergebnisse beim Chronischen Subduralhämatom. Unfallchirurgie 22, 110-116

Putnam TI, Cushing H (1925): Chronic subdural hematoma. Arch Surg 11, 329-393

Rachmandran R, Hegde T (2007): Chronic subdural hematomas- causes of morbidity and mortality. Surg Neurol 67, 367-372 
Reinges MHT, Hasselberg I, Rohde V, Küker W, Gilsbach JM (2000): Prospective analysis of bedside percutaneous subdural tapping for the treatment of chronic subdural hematoma in adults. J Neurol Neurosurg Psychiatry $\underline{69}, 40-47$

Rohde V, Graf G, Hassler W (2002): Complications of burr-hole craniostomy and closedsystem drainage for chronic subdural hematomas: a retrospective analysis of 376 patients. Neurosurg Rev 25, 89-94

Rust T, Kiemer N, Erasmus A (2006): Chronic subdural hematomas and anticoagulation or anti-thrombotic therapy. J Clin Neurosci $\underline{13}, 823-827$

Rychlicki F, Recchioni MA, Burchianti M, Marcolini P, Messori A, Papo I (1991): Percutaneous twist-drill craniostomy for the treatment of chronic subdural hematoma. Acta Neurochir (Wien) 113, 38-41

Santarius T, Kirkpatrick PJ, Ganesan D, Chia HL, Jalloh I, Smielewski P, Richards HK, Markus H, Parker RA et al. (2009): Use of drains versus no drains after burr-hole evacuation of chronic subdural haematoma: a randomised controlled trial. Lancet $\underline{374}$, 1067-1073

Smely C, Madlinger A, Scheremet R (1997): Chronic subdural hematoma- a comparison of two different treatment modalities. Acta Neurochir (Wien) 139, 818-826

Stanisic M, Lund-Johansen M, Mahesparan R (2005): Treatment of chronic subdural hematoma by burr-hole craniostomy in adults: influence of some factors on postoperative recurrence. Acta Neurochir (Wien) 147, 1249-1257

Stanisic M, Aesen AO, Pripp AH, Lindegaard KF, Ramm-Pettersen J, Lyngstadaas SP, Ivanovic J, Konglund A, Ilstad E, Sandell T, Ellingsen O, Saehle T (2012 a): Local and systemic pro-inflammatory and anti-inflammatory cytokine patterns in patients with chronic subdural hematoma: a prospective study. Inflamm Res $\underline{61}, 845-852$

Stanisic M, Lyngstadaas SP, Pripp AH, Aasen AO, Lindegaard KF, Ivanovic J, Ilstad E; Konglund A, Sandell T, Ellingsen O, Saehle T (2012 b): Chemokines as markers of local inflammation and angiogenesis in patients with chronic subdural hematoma: a prospective study. Acta Neurochir (Wien) 154, 113-120 
Stanisic M, Hald J, Rasmussen A, Pripp AH, Ivanovic J, Kolstad F, Sundseth J, Züchner M, Lindegaard KF (2013): Volume und density of chronic subdural hematoma obtained from CT imaging as predictors of postoperative recurrence: a prospective study of 107 operated patients. Acta Neurochir (Wien) 155, 323-333

Steimle R, Jacquet G, Godard J, Fahrat O, Katranji H (1990): Chronic subdural hematoma in the elderly and computerized tomographie. Study of 80 cases. Chirurgie 116, 160-167 (Artikel auf Französisch)

Tabaddor K, Shulmon K (1977): Definitive treatment of chronic subdural hematoma by twist-drill craniostomy and closed-system drainage. J Neurosurg 46 , 220-226

Tahsim-Oglou Y, Beseoglu K, Hänggi D, Stummer W, Steiger HJ (2012): Factors Predicting Recurrence of chronic subdural hematoma: the influence of intraoperative irrigation and lowmolecular weight heparin thromboprophylaxis. Acta Neurochir 154, 1063-1068

Takeda N, Sasaki K, Oikawa A, Aoki N, Hori T(2006): A new simpler therapeutic method for chronic subdural hematoma without irrigation and drainage. Acta Neurochir (Wien) $\underline{143}$, 613-618

Torihashi K, Sadamasa N, Yoshida K, Narumi O, Chin M, Yamagata S (2008): Independent Predictors for Recurrence of Chronic Subdural Hematoma: a Review of 343 Consecutive Surgical Cases. Neurosurgery $\underline{63}, 1125-1129$

Trotter W (1914): Chronic subdural hemorrhage of traumatic origin and its relation to pachymeningitis haemorrhagice interna. Brit J Surg 2 , 271

Tsutsumi K, Maeda K, Iijma A, Usui M, Okada Y, Kirino T (1997): The relationship of preoperative magnetic resonance imaging findings and closed system drainage in the recurrence of chronic subdural hematoma. J Neurosurg $\underline{87}$, 870-875

van den Doel EM (1986): „Balzac`s Pierrette“. An early description of chronic subdural hematoma. Arch Neurol 43, 1291-1292 
van Havenbergh T, Van Calenbergh F, Goffin J, Plets C (1996): Outcome of chronic subdural haematoma: analysis of prognostic factors. Br J Neurosurg $\underline{10}$, 35-39

Virchow R (1857): Das Hämatom der Dura mater. Verhandl Physik Med Gesellsch Würzburg $\underline{7}, 134-142$

Wecht DA (2000): A brief history of chronic subdural hematomas. Neurosurg Clin N Am, $\underline{11}, 395-398$

Weigel R, Schmiedek P, Krauss JK (2003): Outcome of contemporary surgery for chronic subdural haematoma: evidence based review. J Neurol Neurosurg Psychiatry $\underline{74}$, 937-943

White M, Mathieson CS, Campbell E, Lindsay KW, Murray L (2010): Treatment of chronic subdural hematomas- a retrospective comparison of minikraniectomy versus burr hole drainage. Br J Neurosurg $\underline{24}$, 257-260

Yadav YR, Yadav S, Parihar VS (2013): Modified twist drill technique in the management of chronic subdural hematoma. Turkish Neurosurgery $\underline{23}, 50-54$

Yasuda CL, Morita ME, Nishimori FY, Yasuda AM, Alves HL (2003): Chronic subdural hematoma: study of 161 patients and the relationship with coagulation abnormalities. Arq Neuropsiquiatr $\underline{61}, 1011-1014$

Zhang J, Liu Z, Hou RG (2012): Treatment of chronic subdural hematoma by minimally invasive drilling and drainage. Zhonghua Yi Xue Za Zhi 92: 203-205

Zumkeller M, Hollerhage HG, Dietz H (1997): Treatment outcome in patients with chronic subdural hematoma with reference to age and concurrent internal diseases. Wien Med Wochenschr $147,55-62$ 


\section{Danksagung}

Herrn Prof. Dr. med. V. Rohde danke ich für die Überlassung meines Arbeitsthemas, die gute Betreuung, Unterstützung und Übernahme der Erstbegutachtung.

Ein besonderer Dank geht ebenfalls an Herrn PD Dr. Schatlo für die herausragende Unterstützung im Bereich der statistischen Auswertung der erhobenen Daten. 